\title{
Microglia Elimination Increases Neural Circuit Connectivity and Activity in Adult Mouse Cortex
}

\author{
Yong-Jun Liu, ${ }^{1,2}$ Elizabeth E. Spangenberg, ${ }^{3,4}$ Bryan Tang, ${ }^{1}$ 'Dodd C. Holmes, ${ }^{5,6}$ Kim N. Green, ${ }^{3,4}$ and \\ (i) Xiangmin $\mathrm{Xu} \mathbf{u}^{1,4,6}$ \\ ${ }^{1}$ Department Anatomy \& Neurobiology, School of Medicine, University of California, Irvine, California 92697, ${ }^{2}$ Key Laboratory of Pollinating Insect \\ Biology, Institute of Apicultural Research, Chinese Academy of Agricultural Sciences, Beijing, 100093, China, ${ }^{3}$ Department of Neurobiology and \\ Behavior, University of California, Irvine, California 92697, ${ }^{4}$ Institute for Memory Impairments and Neurological Disorders, University of \\ California, Irvine, California 92697, ${ }^{5}$ Department of Physiology and Biophysics, School of Medicine, University of California, Irvine, California \\ 92697, and ${ }^{6}$ Center for Neural Circuit Mapping, University of California, Irvine, California 92697
}

Microglia have crucial roles in sculpting synapses and maintaining neural circuits during development. To test the hypothesis that microglia continue to regulate neural circuit connectivity in adult brain, we have investigated the effects of chronic microglial depletion, via CSF1R inhibition, on synaptic connectivity in the visual cortex in adult mice of both sexes. We find that the absence of microglia dramatically increases both excitatory and inhibitory synaptic connections to excitatory cortical neurons assessed with functional circuit mapping experiments in acutely prepared adult brain slices. Microglia depletion leads to increased densities and intensities of perineuronal nets. Furthermore, in vivo calcium imaging across large populations of visual cortical neurons reveals enhanced neural activities of both excitatory neurons and parvalbumin-expressing interneurons in the visual cortex following microglia depletion. These changes recover following adult microglia repopulation. In summary, our new results demonstrate a prominent role of microglia in sculpting neuronal circuit connectivity and regulating subsequent functional activity in adult cortex.

Key words: excitatory; perineuronal nets; plasticity; parvalbumin; synaptic connections; visual cortex

Significance Statement

Microglia are the primary immune cell of the brain, but recent evidence supports that microglia play an important role in synaptic sculpting during development. However, it remains unknown whether and how microglia regulate synaptic connectivity in adult brain. Our present work shows chronic microglia depletion in adult visual cortex induces robust increases in perineuronal nets, and enhances local excitatory and inhibitory circuit connectivity to excitatory neurons. Microglia depletion increases in vivo neural activities of both excitatory neurons and parvalbumin inhibitory neurons. Our new results reveal new potential avenues to modulate adult neural plasticity by microglia manipulation to better treat brain disorders, such as Alzheimer's disease.

\section{Introduction}

Microglia are the primary immune cells of the mammalian CNS. These cells guard and protect our brains from invading pathogens, akin to macrophages in peripheral organs. It is becoming

\footnotetext{
Received Aug. 13, 2020; revised Nov. 16, 2020; accepted Dec. 13, 2020.

Author contributions: X.X. and K.N.G designed research; Y.L., E.E.S., B.T., T.C.H., K.N.G., and X.X. performed research; Y.L., E.E.S., T.C.H., K.N.G., and X.X. analyzed data; Y.L., E.E.S., K.N.G., and X.X. wrote the paper; B.T. and T.C.H. edited the paper.

This work was supported by National Institutes of Health Grants 1R01NS083801 (National Institute of Neurological Disorders and Stroke) and RF1AG056768 (National Institute on Aging) to K.N.G., R01EY028212 to X.X., and R35GM127102 to T.C.H.

The authors declare no competing financial interests.

Correspondence should be addressed to Kim N. Green at kngreen@uci.edu or Xiangmin Xu at xiangmin.xu@uci.edu.

https://doi.org/10.1523/JNEUROSCI.2140-20.2020

Copyright $(2) 2021$ the authors
}

increasingly clear that microglia play numerous roles outside of immunity, including synaptic remodeling and maintaining brain homeostasis, particularly during development (Nayak et al., 2014; Colonna and Butovsky, 2017). In the developing mouse retinogeniculate system (postnatal days 5-15 [P5-P15]), microglia contribute to activity- and complement-dependent pruning by phagocytosing presynaptic inputs (Stevens et al., 2007; Schafer et al., 2012). During the second and third postnatal weeks in the mouse hippocampus development, microglia engulf synaptic components and contribute to synaptic pruning and maturation (Paolicelli et al., 2011). Mice lacking the chemokine receptor $\mathrm{Cx} 3 \mathrm{cr} 1$ exhibit a transient reduction of microglia during P8-P28 and show impaired synaptic and neural network connectivity and abnormal social behavior in adulthood (Paolicelli et al., 2011; Zhan et al., 2014). Other structural and physiological evidence also supports that microglia 
modulate excitatory glutamatergic signaling at both presynaptic and postsynaptic sites, resulting in changes in synaptic connectivity during development (Schafer et al., 2012; Ji et al., 2013).

Microglia undergo morphological, phenotypical, and cell density changes during postnatal brain development. Ramified microglial processes constantly modify connections with synapses (Nimmerjahn et al., 2005; Tremblay et al., 2011). C1q is a complement cascade protein; its activation triggers synapse elimination by microglia. Mice genetically lacking $\mathrm{Clq}$ show excessive retinal innervation of lateral geniculate neurons $(\sim \mathrm{P} 30)$, and enhanced excitatory synaptic connectivity and epileptiform activity (P19-P39) in sensorimotor cortex (Stevens et al., 2007; Chu et al., 2010). EPSC frequency increases in cultured hippocampal brain slices with microglia depleted by clodronate liposomes; subsequently, the addition of microglia to neuronal cultures decreases synaptic activity (Ji et al., 2013).

Our prior work suggests that microglia continue to regulate neuronal structures in the adult brain. Microglia in the adult brain depend on signaling through the colony-stimulating factor 1 receptor (CSF1R) (Elmore et al., 2014). Administration of selective CSF1R inhibitors that cross the blood-brain barrier rapidly eliminate $>95 \%$ of all microglia in the CNS, and suppress microglia for as long as treatment is maintained. Brain microglia levels recover after the inhibitor treatment ceases (Elmore et al., 2014; Spangenberg et al., 2019). Using timed administration of CSF1R inhibitors, we find that adult mice lacking microglia show remarkable elevations in the densities of dendritic spines (the sites of excitatory synapses between neurons) in all brain regions surveyed, along with increases in synaptic puncta (Elmore et al., 2014; Rice et al., 2015, 2017). Conversely, aberrant synaptic loss through over-pruning is a key feature of many diseases characterized by overly reactive microglia, such as Alzheimer's disease (Spangenberg et al., 2016, 2019; Rajendran and Paolicelli, 2018). Chronic microglial depletion in the mouse model of $\mathrm{AD}$ rescues dendritic spine loss and prevents neuronal loss in the brain (Spangenberg et al., 2016), as well as prevents extensive loss of perineuronal nets (PNNs) (Crapser et al., 2020b). Similar results with PNNs have been found in mouse models of Huntington's disease (Crapser et al., 2020a). Related to these changes in PNNs, a recent study reports that microglial remodeling of the extracellular matrix promotes synapse plasticity in adult hippocampus (Nguyen et al., 2020). Thus, it is important to further understand whether and how microglia regulate neuronal connections in normal and diseased adult brains.

In the present study, we investigate the effects of chronic microglial depletion via CSF1R inhibitors on neuronal structure and synaptic connectivity in the visual cortex in normal adult mice. We find that microglia depletion in adult cortex induces robust increases in PNNs, and enhances local excitatory and inhibitory circuit connectivity to excitatory neurons. Compared with control, microglia depletion increases neural activities of both excitatory neurons and parvalbumin (PV) inhibitory neurons. Our new data demonstrate a prominent role of microglia in sculpting neuronal circuit connectivity and regulating subsequent functional activity in adult cortex.

\section{Materials and Methods}

Animal treatments. All mouse experiments were performed in accordance with animal protocols approved by the Institutional Animal Care and Use Committee at the University of California, Irvine. To deplete microglia, mice were treated with the CSF1R/c-Kit inhibitor PLX3397 (3 weeks; 290 ppm in Ain76A chow) (Elmore et al., 2014) or the more specific CSF1R inhibitor PLX5622 (5 weeks; $1200 \mathrm{ppm}$ in Ain76A chow) starting at the age of $8-10$ weeks (Spangenberg et al., 2016, 2019). The two complementary CSF1R inhibitors were used; this minimized the possibility of any nonspecific effects of either inhibitor. The effects of PLX5622 and PLX3397 on the neural activities appeared to be identical. Therefore, we only used PLX3397 for most experiments in the paper. Both inhibitors were provided by Plexxikon and formulated in standard chow by Research Diets in Ain76A chow. The treatment duration of PLX compounds is specified in the descriptions of individual experiments.

Mice were randomly assigned to experimental versus control groups with matched age and sex. Experiments were repeated, and the results were reproducible. Experimenters were not blinded during data acquisition, but imaging data analyses were performed blind to treatment.

Histology, immunofluorescent staining, and image acquisition. Animals were transcardially perfused under deep isoflurane anesthesia, and brains were left in 4\% PFA for overnight fixation. Samples were switched into $30 \%$ sucrose in PBS. Brain samples were then coronally sectioned into $30-\mu \mathrm{m}$-thick samples using a freezing microtome (Leica Microsystems, SM2010R), secured to a frozen sucrose base. Fluorescent immunolabeling followed a standard protocol (primary antibody followed by fluorescent secondary antibody), as previously described (Xu et al., 2010a; Elmore et al., 2014).

To assess the effects of PLX treatments, we immunochemically stained selected brain sections to label microglial populations. Samples were washed with $1 \times$ PBS and placed on an orbital shaker for $5 \mathrm{~min}$ for 3 times. Sections are transferred into blocking medium $(0.075 \%$ Triton-X, $5 \%$ normal donkey serum in $1 \times$ PBS solution) for $2 \mathrm{~h}$ at room temperature before applying primary antibody. Primary antibodies used include the following: NeuN (1:1000; Abcam; RRID:AB_1566475), IBA1 (1:1000; Wako; RRID: AB_839506), GFAP (1:10,000; Abcam; RRID:AB_296804), Wisteria floribunda agglutinin (WFA, 1:1000; Vector Labs; RRID:AB_2336875), PV (1:1000, Swant; RRID:AB_2313848), and S100 $\beta$ (1:1000, Abcam; RRID:AB_991821). Primary antibodies were applied for $48 \mathrm{~h}$, and followed by AF488-, Cy3-, or Cy5-conjugated appropriate secondary antibodies (Jackson ImmunoResearch Laboratories, 1:200). Sections were counterstained with $10 \mu \mathrm{M}$ DAPI, then mounted and coverslipped. Immunostained sections in Figure 1 were examined using a confocal microscope (LSM 780, Carl Zeiss) with $10 \times$ image stacks. Image tiles, overlaying, maximum projections, and subset $z$-stack selections were performed using the Carl Zeiss image processing software.

Numbers and signal intensity of microglia, astrocytes, PV, and PNNs were obtained by scanning regions at $10 \times$ at comparable sections in each animal, the scanning regions were the FOVs, and then numbers of microglia were measured automatically using the spots module in Bitplane Imaris 7.5 software. We used $>20$ FOVs from at least 3 animals for each condition for quantifications in Figure 1.

Electrophysiological recording and analyses. Electrophysiological recordings and photostimulation were performed as extensively described previously (Xu and Callaway, 2009; Xu et al., 2010b, 2016; Olivas et al., 2012; Kuhlman et al., 2013). Six-week-old mice were treated for 3-4 weeks with control chow ( $n=12$ mice), or PLX3397-formulated chow ( $n=10$ mice) or PLX5622-formulated chow ( $n=4$ mice) to deplete microglia. Animals were deeply anesthetized using pentobarbital sodium $(100 \mathrm{mg} / \mathrm{kg}$, i.p.). Brains were extracted; coronal sections of visual cortical of $400 \mu \mathrm{m}$ were made with a vibratome (VT1200S, Leica Microsystems) in sucrose containing ACSF (in mM as follows: $85 \mathrm{NaCl}, 75$ sucrose, $2.5 \mathrm{KCl}, 25$ glucose, $1.25 \mathrm{NaH}_{2} \mathrm{PO}_{4}, 4 \mathrm{MgCl}_{2}, 0.5 \mathrm{CaCl}_{2}$, and 24 $\mathrm{NaHCO}_{3}$ ). Slices were incubated for at least $30 \mathrm{~min}$ in normal ACSF (in mM as follows: $126 \mathrm{NaCl}, 2.5 \mathrm{KCl}, 26 \mathrm{NaHCO}_{3}, 2 \mathrm{CaCl}_{2}, 2 \mathrm{MgCl}_{2}, 1.25$ $\mathrm{NaH}_{2} \mathrm{PO}_{4}$, and 10 glucose) at $32^{\circ} \mathrm{C}$ before transfer into slice recording chambers. Throughout the cutting, incubation, and recording, the solutions were continuously supplied with $95 \% \mathrm{O}_{2}-5 \% \mathrm{CO}_{2}$.

Whole-cell recordings were performed under a differential interference contrast/fluorescent Olympus microscope (BX51WI) at room temperature. Slices were examined under a $4 \times$ objective for proper targeting of layer 5 V1 neurons. To perform whole-cell recording, individual neurons were visualized at high magnification $(60 \times$ objective, $0.9 \mathrm{NA}$; 

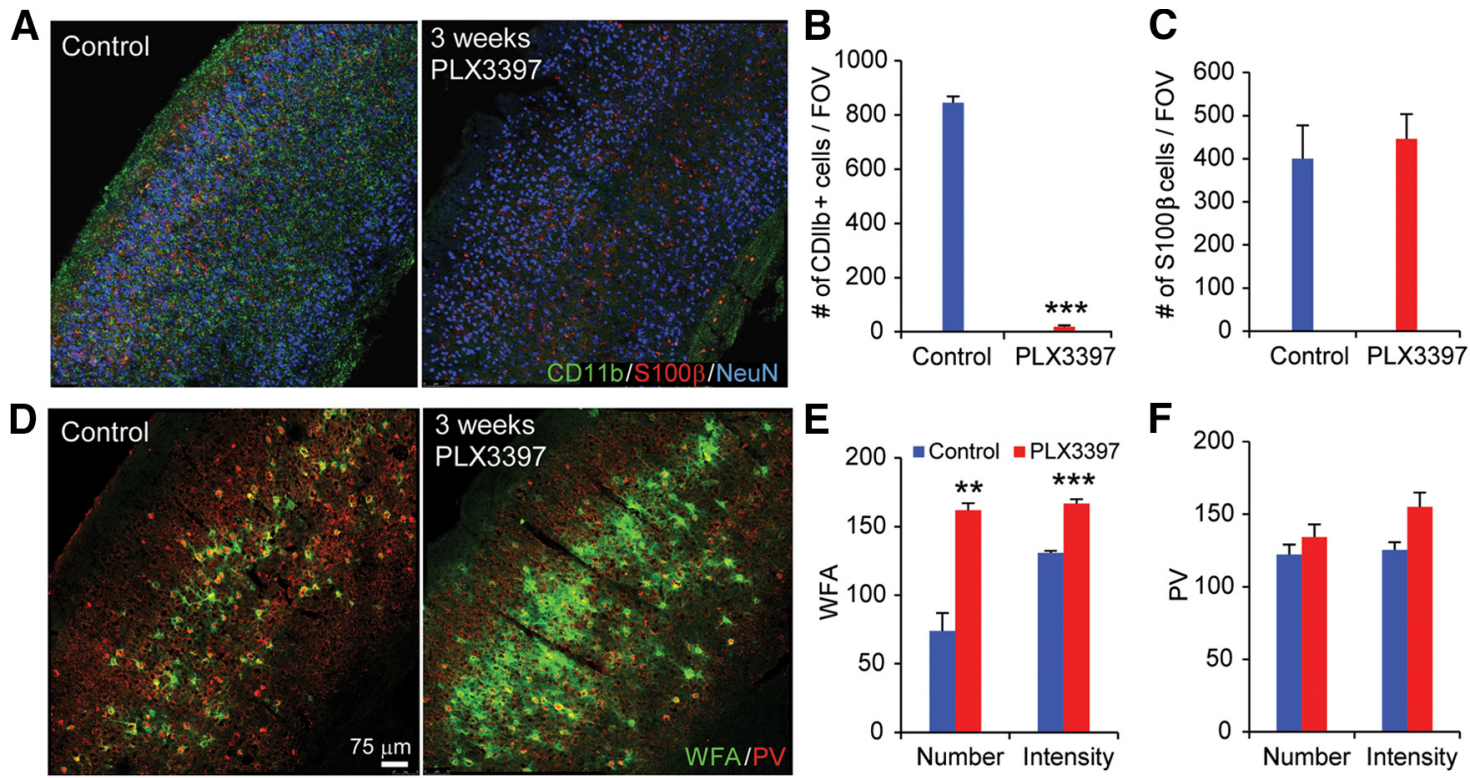

$\mathbf{F}$
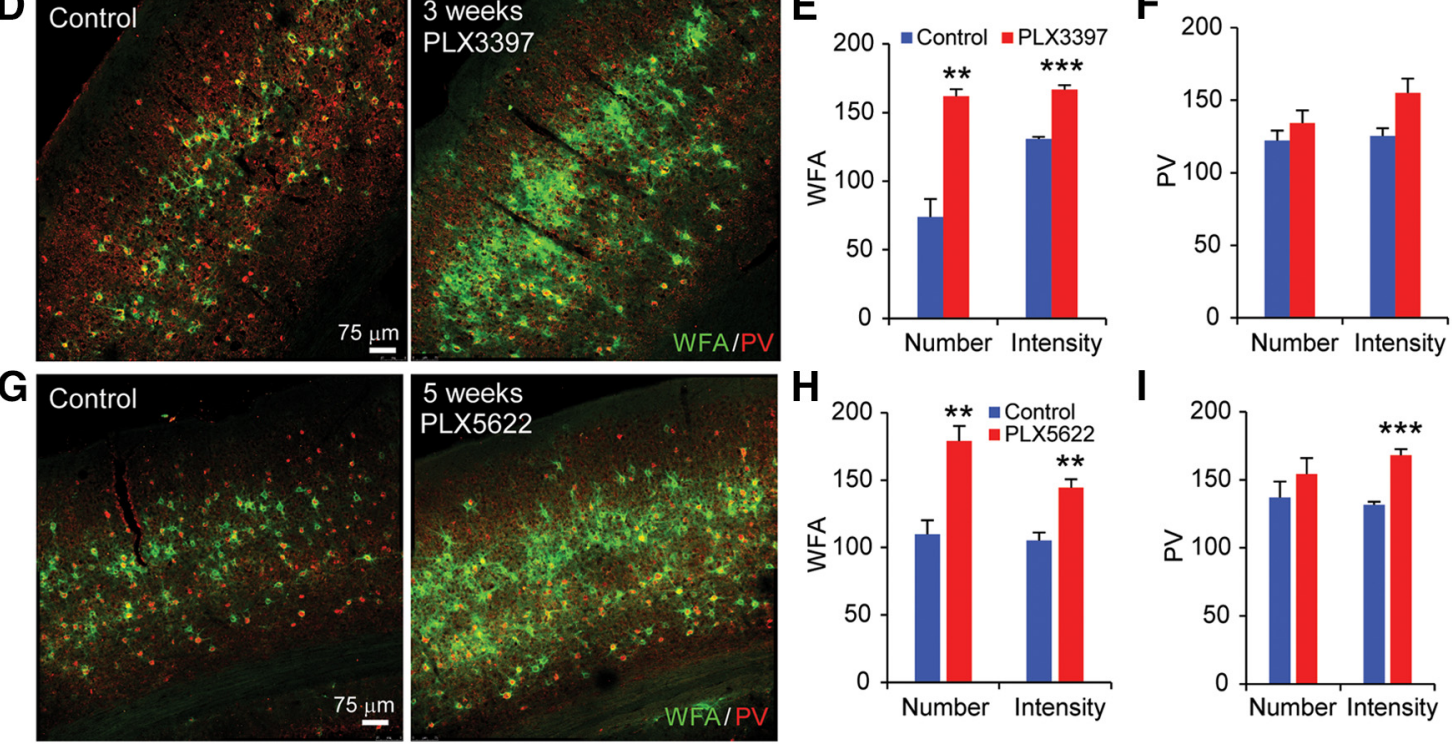

Figure 1. Microglial depletion by treatment with CSF1R inhibitors PLX3397 and PLX5622 robustly enhances PNNs. A, WT C57BL6/J mice treated for 3 weeks with control diet, or PLX3397 (290 ppm in chow) or PLX5622 (1200 ppm in chow). CD11b, S100 $\beta$, and NeuN staining in visual cortex sections is shown for control (left) and PLX3397 treatment of 3 weeks (right). $\boldsymbol{B}$, Numbers of CD11 $\mathrm{b}^{+}$cells/FOV are plotted with control and PLX3397 treatment. Microglia are reduced by $\sim 95 \%$ with this PLX3397 treatment $(p=0.001)$. C, Quantification of the number of S100 $\beta^{+}$cells/FOV for control and PLX3397 conditions showing that astrocyte intensities were unaffected by PLX3397. D, WFA and PV staining is shown for control condition (left) and for PLX3397 treatment (right). $\boldsymbol{E}$, Quantification shows that the numbers and intensity of WFA-positive PNNs per FOV significantly increase following 3-week-long PLX3397 treatment ( $p=0.003$ and $\left.p=5.56 \times 10^{-4}\right)$. $F$, The PLX3397 treatment does not modulate the number and intensity of PV neurons $(p=0.335$ and $p=0.056)$. G, WFA and PV staining following 5-week-long PLX5622 treatment. WFA and PV numbers and intensities for each condition are plotted in $\boldsymbol{H}$ and $\boldsymbol{I}$. WFA is significantly increased with PLX5622 treatment $(p=0.002$ for WFA number; and $p=0.002$ for WFA intensity). Although the numbers of PV neurons are not affected $(p=0.359)$, PV staining intensity is significantly increased with PLX5622 treatment $\left(p=2.93 \times 10^{-4}\right)$. Data are mean \pm SEM (samples from at least 3 mice/group). $* * p \leq 0.01 ; * * * p \leq 0.001$; two- tailed unpaired Student's $t$ test.

LUMPIanFI/IR, Olympus America), and patched with borosilicate glass electrodes of 4-6 $\mathrm{M} \Omega$ resistance filled with $\mathrm{K}^{+}$-containing internal solution (126 mm K-gluconate, $4 \mathrm{~mm} \mathrm{KCl,} 10 \mathrm{~mm}$ HEPES, $4 \mathrm{~mm}$ ATP-Mg, $0.3 \mathrm{~mm}$ GTP-Na, and $10 \mathrm{~mm}$ phosphocreatine, $\mathrm{pH}$ 7.2, $300 \mathrm{mOsm}$ ) when measuring EPSCs. While recording inhibitory postsynaptic currents IPSCs, a cesium-based internal solution ( $130 \mathrm{~mm} \mathrm{CsOH}, 130 \mathrm{~mm}$ D-gluconic acid, 0.2 mм EGTA, 2 mм $\mathrm{MgCl}_{2}, 6 \mathrm{~mm} \mathrm{CsCl,} 10$ mм HEPES, 2.5 mм ATP-Na, $0.5 \mathrm{~mm}$ GTP-Na, and $10 \mathrm{~mm}$ phosphocreatine, $\mathrm{pH} 7.2,300 \mathrm{mOsm}$ ) was used. Electrodes also contain $0.1 \%$ biocytin for post hoc cell labeling and further morphologic identification. Once stable, whole-cell recordings were achieved with good access resistance $(<30 \mathrm{M} \Omega)$, and basic electrophysiological properties were examined through hyperpolarizing and depolarizing current injections. Electrophysiological data were acquired by a custom-modified version of Ephus software. Resting membrane potentials, input resistance, and membrane capacitance, as well as spiking patterns, were all measured. To measure spontaneous excitatory postsynaptic currents (EPSCs), the recorded neuron was held at $-70 \mathrm{mV}$ in voltage-clamp mode, and the data of spontaneous traces were acquired over $10 \mathrm{~min}$.

During laser scanning photostimulation (LSPS) experiments, the microscope objective was switched from $60 \times$ to $4 \times$. Stock solution of MNI-caged-L-glutamate (Tocris Bioscience) was added to $20 \mathrm{ml}$ of ACSF for a concentration of $0.2 \mathrm{~mm}$ caged glutamate. The cortical slice image was acquired by a high-resolution digital CCD camera, which in turn was used for guiding and registering photostimulation sites. Using an electro-optical modulator, short pulses of laser flashes (1 ms, $20 \mathrm{~mW})$ were delivered, which generate the focal laser spots with approximate a Gaussian profile of a lateral width of $100 \mu \mathrm{m}$. Under our experimental conditions, LSPS-evoked action potentials were recorded from stimulation locations within $\sim 100 \mu \mathrm{m}$ of targeted somata and occurred within $150 \mathrm{~ms}$ after photostimulation. Synaptic currents in patched neurons were detected under voltage clamp. By systematically surveying synaptic inputs from hundreds of different sites across a large cortical region, aggregate synaptic input maps were generated for individual neurons.

LSPS allows for extensive and quantitative mapping of local circuit inputs. A mouse V1 coronal slice image was used with the superimposed photostimulation sites $(16 \times 16$, spaced at $60 \mu \mathrm{m} \times 60 \mu \mathrm{m})$ across all the cortical layers. The electrode was recording from an excitatory pyramidal neuron in upper layer 5. During the experiment, the slice was bathed in the solution containing MNI-caged glutamate $(0.2 \mathrm{mM})$, which only turns active through focal UV photolysis to activate a small number of neurons (i.e., glutamate uncaging). The whole cortical mapping field was stimulated sequentially with an interval of $1.2 \mathrm{~s}$ in a nonraster and nonrandom sequence.

Similar to LSPS, we mapped PV specific inhibitory inputs to excitatory neurons using laser scanning channelrhodopsin-2 (ChR2) photoactivation. This was achieved with PV inhibitory cell type-specific optogenetic stimulation. The PV-Cre mouse line was crossed to the Ai32 mouse line (Madisen et al., 2012), which has a conditional allele of RosaCAG-LSL-ChR2(H134R)-EYFP-WPRE, to drive ChR2/EYFP fusion 
protein expression in PV cells. Whole-cell recordings were made from layer 5 pyramidal neurons in visual cortex of PV-Cre;Ai32 mice; spatial maps of PV cell connectivity strength to each patched pyramidal neuron were derived by systematically stimulating ChR2-expressing PV cells at $16 \times 16$ different sites arranged in a mapping grid across all cortical layers. Spatially restricted optogenetic activating of ChR2-expressing PV cells was accomplished using a $473 \mathrm{~nm}$ blue laser $(0.25 \mathrm{~ms}, 3 \mathrm{~mW}$; laser spot diameter, $\sim 50 \mu \mathrm{m})$. Whole-cell voltage-clamp recordings were made from the recorded pyramidal neurons to measure ChR2-evoked inhibitory postsynaptic currents (IPSCs) at the empirically determined holding potential at $5 \mathrm{mV}$ with cesium-containing internal solution (for more detailed information, see Sun et al., 2016).

For photostimulation data analysis, the amplitudes, frequencies, and onset latencies of EPSCs or IPSCs from the recorded cells were measured for each stimulation site using custom-developed MATLAB software (Shi et al., 2010). Laminar input patterns and source distributions were quantified. LSPS-evoked EPSCs were quantified across the $16 \times 16$ mapping grid for each cell, and 2-4 individual maps are averaged to bin responses according to laminar cytoarchitectonic landmarks. The EPSC input from each stimulation site was the measurement of the sum of individual EPSCs within the analysis window (10-160 ms after photostimulation), subtracting baseline spontaneous response from the photostimulation response of the same site. Then it was normalized with the duration of the analysis window and expressed as average integrated amplitudes in picoamperes (pA). Synaptic events were binned from locations spanning $\pm 400 \mu \mathrm{m}$ tangential to the targeted soma location and from the top of layer $2 / 3$ to the bottom of layer 6 across the radial vector. Data were plotted as either the average integrated EPSCs amplitude per pixel location or the number of EPSCs detected per pixel location. The quantitative color-coded map, which measured the EPSC input strength per stimulation, was generated by averaging integrated amplitudes of individual EPSCs at each site in the specified analysis window (within $150 \mathrm{~ms}$ after photostimulation). The ChR2-photoactivation data analysis was similar to that described above for LSPS.

In vivo two-photon calcium imaging. For two-photon in vivo calcium imaging, the GCaMP6s transgenic mouse expressing GCaMP6s in excitatory neurons (Wekselblatt et al., 2016) and PV-Cre;Ai163 mouse (Daigle et al., 2018) expressing GCaMP6s in PV cells were used. The general procedure for surgery has been described previously (Kuhlman et al., 2013; Grieco et al., 2020). After the skull was exposed, it was dried and covered by a thin layer of Krazy glue. After the Krazy glue dried $(\sim 15 \mathrm{~min})$, it provided a stable and solid surface on which to affix an aluminum headplate with dental acrylic. The headplate was affixed to the skull, and the margins were sealed with dental acrylic to prevent infections. A 4-mm-diameter region of skull overlying the occipital cortex was removed. The craniotomy opened at center coordinates $3 \mathrm{~mm}$ lateral, and $1.7 \mathrm{~mm}$ anterior to the lambda suture. Care was taken to leave the dura intact. A sterile, 4-mm-diameter cover glass was then placed directly on the dura and sealed at its edge with Krazy glue. When dry, the edges of the cover glass were further sealed with dental acrylic. At the end of the surgery, all exposed skull and wound margins were sealed with VetBond and dental acrylic. Mice were then removed from the stereotaxic apparatus, given a subcutaneous bolus of warm sterile saline and carprofen $(5 \mathrm{mg} / \mathrm{kg}$, subcutaneous), and allowed the mouse to recover on the heating pad. When fully alert, they were placed back in their home cages. Carprofen was given for 3 consecutive days. Typically, the mouse was permitted to recover for at least $7 \mathrm{~d}$ and conditioned to the head restraint and running wheel for several days for $20 \mathrm{~min}$ per day before experiments.

Two-photon calcium imaging was performed in the binocular region of $\mathrm{V} 1$ by the resonant scanning two-photon microscope system controlled by Scanbox acquisition software (Neurolabware). Retinotopic maps were acquired by GCaMP6-based epifluorescence imaging using a $4 \times, 0.16 \mathrm{NA}$ objective. The surface vasculature and GCaMP6s signals were visualized with illumination of white mounted LEDs (400-700 nm) (MCWHLP1, Thorlabs), via a bandpass filter $(482 / 18 \mathrm{~nm}$, Thorlabs), and fluorescence was detected by a sCMOS camera (PCO edge $4.2 \mathrm{LT}$ ) via a 520/28 nm bandpass filter (Thorlabs). The focal plane of the microscope focused $400-600 \mu \mathrm{m}$ below the surface vasculature during retinotopic mapping. The mouse was awake during retinotopic mapping, the head was restrained via the headplate, and the eyes were on a horizontal plane. The mouse was trained and habituated to a running wheel $3 \mathrm{~d}$ before the imaging. GCaMP6-based epifluorescence signal was stronger than intrinsic signals. Eye movements occurred during all phases of the wide-field mapping stimulus, and the mean eye displacement was $<1$ degree (Zhuang et al., 2017). Furthermore, eye movementrelated effects were largely eliminated during data processing by averaging repetitions of visual stimulation in offline data analysis. Therefore, the global retinotopy mapping was largely unaffected by small eye movements (Zhuang et al., 2017).

We used the same visual stimulation as in Zhuang et al. (2017), which was generated in real time with processing using OpenGL shaders. The stimulation was displayed on a 27 inch LCD monitor (Asus, vg 278) with a refresh rate of $60 \mathrm{~Hz}$, placed perpendicularly at $20 \mathrm{~cm}$ from the mouse. The monitor was extended from $\sim-56$ to 56 degrees in the azimuth and from -40 to 40 degrees in the elevation. A TTL pulse was generated with an Arduino at each stimulus transition, and the pulse was recorded and time-stamped for synchronizing between visual stimulation and imaging data. Retinotopic responses were generated by sweeping a stimulus bar across the monitor (Kalatsky and Stryker, 2003). The bar contained a 4-degree-wide, black-and-white checkerboard pattern, with a gray background of $50 \%$ of the maximum luminance. The stimulus was swept across the screen for 8 times in each of four cardinal directions with a duration of $10 \mathrm{~s}$. There was a $10 \mathrm{~s}$ interval of blank gray screen inserted between sweeps. Azimuth and elevation retinotopic maps were used to generate a visual field sign map (Garrett et al., 2014). Binocular V1 was confined to regions adjacent to the intersection of the horizontal and vertical meridians at the border of V1 and lateromedial area (LM).

For in vivo two-photon calcium imaging, $920 \mathrm{~nm}$ wavelength was used for imaging calcium responses of excitatory and PV neuron types. The microscope objective used was a $16 \times$ water-immersion lens (Nikon, $0.8 \mathrm{NA}, 3 \mathrm{~mm}$ working distance). Usually, we imaged simultaneously from four layers of neurons separated by $20 \mu \mathrm{m}$ in cortical layer $2 / 3$ (100-300 $\mu \mathrm{m}$ below pia) with a bidirectional scanning mode. The image sequences were captured at $8 \mathrm{~Hz}$ for each layer of neurons. The image area covered a field of $\sim 796 \mu \mathrm{m} \times 494 \mu \mathrm{m}$. We examined baseline levels of cortical activity, and the visual display was simply a gray screen of 20 $\mathrm{cd} / \mathrm{m} 2$ luminance shown for $15 \mathrm{~min}$ while collecting calcium imaging data. This experimental design was sufficient to quantify neural activity levels (see Figs. 6 and 7) and avoided introducing artifacts associated with visual experience-dependent plasticity (Kaplan et al., 2016). Eye movements and pupil sizes were recorded via a Dalsa Genie M1280 camera (Teledyne Dalsa) fitted with a UV cutoff filter (wavelength range 350-2200 $\mu \mathrm{m}$ ). During two-photon imaging, a substantial amount of light exited from the skull; no additional illumination was required to image the pupil.

Mice were head-fixed on a running wheel on which they had been trained to balance and run. Movement of the wheel was recorded via a ball-bearing option tracker (H5, US digital). Both locomotion and eye movement data were synchronized to the microscopic image frames. Locomotion was used for excluding calcium response traces falling in running sessions. We had analyzed pupil sizes across conditions and found out that they did not significantly differ. Therefore, there was no response correction to pupil sizes.

In order to longitudinally align the imaging area for each recording, we used blood vessel landmarks and robustly active neurons as reference positions for alignment of the imaging areas. This allowed us to examine the same groups of neurons across sessions.

Analysis for two-photon calcium imaging. Custom-written MATLAB pipelines based on CaImAn, which is an open-source library for calcium imaging data analysis, was used for offline analysis (Giovannucci et al., 2019). The positions of neurons might change slightly because of the cortex movement during imaging. This motion artifact was corrected by NormCorre pipeline (Pnevmatikakis and Giovannucci, 2017). The frames were smoothed with a $12 \times 12$ pixel Gaussian kernel $(S D=4)$ to suppress the background noise; then nonrigid translation was applied to every two successive frames by dividing each frame of multiple $128 \times$ 
128 pixel patches. We estimated the motion effect between corresponding patches and translated them for alignments. After removing motion artifact, we aligned each imaging session with several robust neurons as references to correct for translation and rotation differences between two imaging sessions. After motion correction and footprint alignments, temporal calcium traces of neurons were extracted from the recording using the Constrained Nonnegative Matrix Factorization-based CalmAn pipeline. The pipeline models the calcium signal with the following equation:

$$
Y=\mathrm{aC}+b+e .
$$

Where $Y$ is the observed calcium recording, $a$ is the matrix that contains the "spatial footprint" of each neuron soma, $C$ is the matrix that contains each neuron's calcium dynamic, $b$ is the background, and $e$ is the Gaussian noise matrix that compensates the estimation errors. The initializing of $a, C, b$, and $e$ uses the greedy ROI method described by Pnevmatikakis et al. (2016). Then the Constrained Nonnegative Matrix Factorization was iteratively applied to update and refine the spatial footprint and temporal calcium signals. The neuropil signal is contained in $B$ and discarded after the processing. The amplitudes of calcium responses $(\Delta \mathrm{F})$ were subtracted by baseline responses, and were converted to $\Delta \mathrm{F} / \mathrm{F}$ using the following steps: (1) the baseline fluorescence response (F0) of each neuron is estimated by $Y i-a i \times C i$. The original calcium trace of the footprint is $Y i$, its spatial footprint is $a i$, and the extracted temporal calcium trace is $\mathrm{Ci}$. (2) And then calculate mean F0, which is F0_avg. (3) $\Delta \mathrm{F} / \mathrm{F}=C i / \mathrm{F} 0 \_$avg. For the extracted calcium events of each neuron, the peak event amplitude was the closet peak amplitude of the $\Delta \mathrm{F} / \mathrm{F}$ signals. Calcium response events that exceeded a threshold of $10 \%$ of the maximum event amplitude across the recording duration were included for further analysis. The calcium event frequency was calculated by normalizing the total number of detected events within the session length (15 min each). For our analysis in this study, only nonrunning episodes were included for analysis. All extracted neurons from each imaging session were included in the analysis.

Statistics. For immunochemical staining data analysis in Figure 1, two-tailed unpaired Student's $t$ test was used to analyze differences between control and PLX treatment groups of mice. Results are presented as raw mean \pm SEM. Significance was defined as $p<0.05$. For electrophysiological data analysis (Figs. 2-5), statistical comparisons of the measurements across groups were made using the Kruskal-Wallis test, and Mann-Whitney $U$ tests for group comparisons. Alpha levels of $p \leq 0.05$ were considered significant.

For two-photon data analysis, we used the Kruskal-Wallis test and Mann-Whitney $U$ test for comparing multiple day responses with the first-day baseline response. Alpha levels of $p \leq 0.05$ were considered significant. Furthermore, to confirm that the changes we observed across control chow and PLX5622/PLX3397 treatments were better accounted for by groups than differences between individual animals, we analyzed data with a linear mixed-effects model using treatments as a fixed effect, and mouse identity as a random effect. The linear mixed-effects model has been increasingly used to analyze correlated data, such as longitudinal data and clustered data, including longitudinal two-photon calcium imaging data (Stobart et al., 2018a,b; Moda-Sava et al., 2019). The comparisons in Figures 6 and 7 involved multiple comparisons. To limit the potential high false discovery rate caused by multiple testing, we corrected the original $p$ values using the Benjamini-Hochberg procedure, and reported for multiple comparisons.

\section{Results}

Microglial depletion increases PNNs in the visual cortex

We have previously defined local excitatory and inhibitory neural circuits in the adult mouse visual cortex (Kuhlman et al., 2013; Sun et al., 2016; Xu et al., 2016), and have shown increased dendritic spine densities of excitatory cells in layer 5 following microglial depletion in the same region (Rice et al., 2015). To extend these studies, we set out to define the roles of microglia in regulating neural connectivity in the mouse visual cortex. We discovered in our previous work that inhibiting CSF1R signaling leads to microglial depletion (Green et al., 2020). To confirm microglial depletion following CSF1R inhibition, we stained for the microglia marker CD11b, and measured confocal images of the visual cortex by automated cell counts. We found a $\sim 95 \%$ reduction in microglial numbers after 3 weeks of CSF1R inhibitor treatment (Fig. 1A,B; PLX3397-treated mice shown). Immunostaining for $\mathrm{S} 100 \beta^{+}$astrocytes reveals no changes in astrocyte cell numbers, or $\mathrm{S} 100 \beta$ staining intensity, with treatment (Fig. 1A,C). As a morphologic surrogate for plasticity, we visualized PNNs with WFA, a well-established and highly sensitive marker that binds to terminal $\mathrm{N}$-acetylgalactosamine of chondroitin sulfates (Hartig et al., 1992). PNNs are extracellular matrix structures associated with changes in neural plasticity that surround certain neuronal subtypes (Carulli et al., 2010; Colonna and Butovsky, 2017), and are primarily composed of chondroitin sulfate proteoglycans of the aggrecan family, as well as hyaluronic acid, and tenascin-R (Brückner et al., 2000). Robust increases in both PNN density and intensity are seen with microglial depletion, with either PLX3397 (Fig. 1D,E; PLX3397 vs control, $p=0.003$ and $p=5.56 \times 10^{-4}$ ) or PLX5622 (Fig. $1 G, H$; PLX5562 vs control $p=0.002$ and $p=0.002$ ). Further, we visualized PV, a marker of PV-expressing inhibitory neurons as its expression is regulated by neuronal activity (Donato et al., 2013). Notably, mean PV staining intensities increase concomitantly with time of microglial depletion and reach significance at 5 weeks after treatment of PLX5622 relative to age-matched untreated controls (Fig. $1 I ; p=2.93 \times 10^{-4}$ ). Together, depletion of microglia leads to the elevation of numbers and intensities of PNNs following treatment by either of the CSF1R inhibitors (PLX3397, PLX5622), and coincides with increased PV staining with 5-week-long treatment of PLX5622.

\section{Microglial depletion enhances excitatory connectivity in the visual cortex}

As microglial depletion increases PNNs and PV expression in the adult visual cortex, we next conducted functional circuit mapping experiments in living visual cortical slices. We treated 6-week-old mice with either control chow or PLX3397 for 34 weeks to deplete microglia, and acute brain slices were prepared at 10 weeks of age (Fig. 2A). Microglial depletion is confirmed after electrophysiological recordings via immunostaining for the microglial marker IBA1 (Fig. $2 A$; the recorded neuron is also shown by biotin filling [red]). Whole-cell recordings were obtained from V1 layer 5 excitatory neurons of control mice and PLX3397-treated mice (example recordings shown in Fig. 2A,B); intrinsic membrane properties, including average input resistance (input R), membrane capacitance, and membrane resistance, as well as general spiking patterns of excitatory neurons recorded in control and microglia-depleted mouse brain slices, are not significantly different from one another (Fig. $2 C, D$ ). In accordance with prior data in microglia-depleted hippocampal brain slice cultures using clodronate liposomes (Ji et al., 2013), these results show that, under normal conditions, neither microglia nor CSF1R inhibition directly regulates intrinsic membrane properties of cortical neurons.

As microglia contribute to synaptic remodeling and plasticity (Paolicelli et al., 2011; Schafer et al., 2012; Nguyen et al., 2020), we examined the synaptic activity of recorded V1 layer 5 excitatory pyramidal cells in control and PLX3397-treated mice. Interestingly, spontaneous EPSC frequencies increase significantly in recorded excitatory neurons in microglia-depleted brain slices compared with those from the control mice (Fig. 2E), 
A
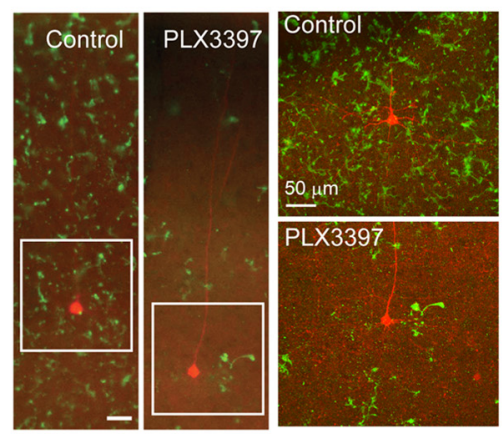

C

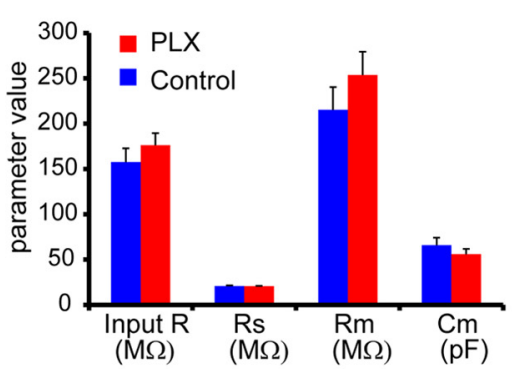

B

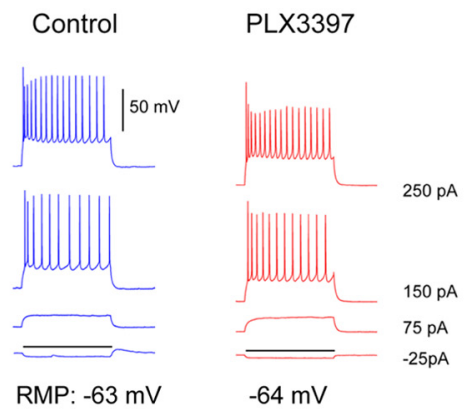

D

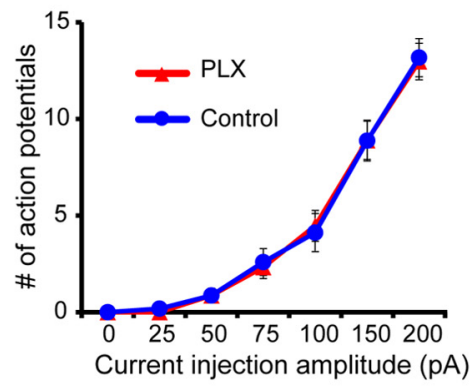

E Spontaneous EPSCs

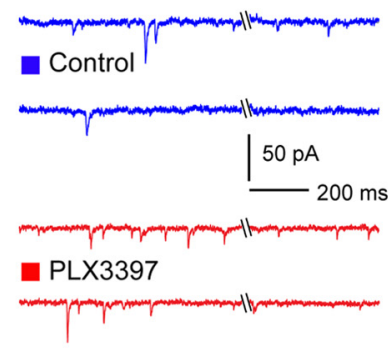

$\mathbf{F}$

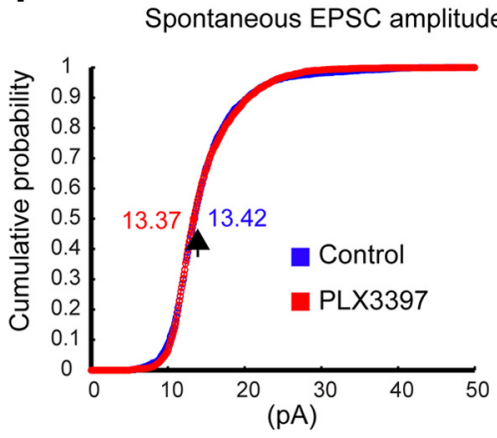

G

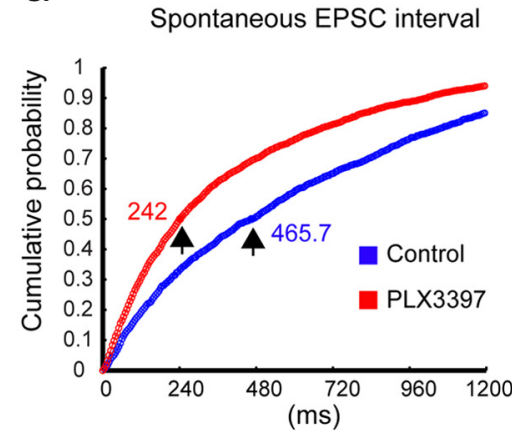

Figure 2. Spontaneous EPSCS increase substantially while intrinsic electrophysiological properties of excitatory pyramidal cells are not changed following 3- to 4-week-long PLX3397 treatment. $A$, Example cortical slice images of mice on PLX3397 show effective depletion of microglia cells, compared with control. The slices were postfixed after physiological recordings and immunostained against the microglia marker, IBA1. The recorded excitatory cells are visualized with biocytin staining (red); green represents microglial cells. Scale bar, $50 \mu \mathrm{m} . \mathbf{B}$, Hyperpolarized and depolarized responses of the representative V1 layer 5 excitatory pyramidal cells in response to intrasomatic current injections. $C$, Comparison of average input resistance (input $R$ ), series resistance $\left(R_{s}\right)$, membrane resistance $\left(R_{m}\right)$, and membrane capacitance $\left(C_{m}\right)$ between excitatory pyramidal cells of mice on PLX3397 ( $n=12$ cells from 4 C57BL/6 mice) and control $(n=12$ cells from 3 (57BL/6 mice). Data are mean \pm SEM. $\boldsymbol{D}$, The current injection-response functions do not differ between the recorded cells from PLX3397-treated and control brain slices. $\boldsymbol{E}$, Example traces showing spontaneous EPSCs from two representative neurons in control and PLX3397-treated mice. $\boldsymbol{F}$, Cumulative probability plots of spontaneous EPSC amplitude distribution in control (blue curve) and PLX3397 (red curve) for 6 cells each. The cumulative probability curves overlap (Mann-Whitney $U$ test; $p=0.796$ ), and the amplitudes at the 0.5 cumulative probability essentially are the same (13.42 vs $13.37 \mathrm{pA}$ ) between control and PLX treatment. G, Cumulative distribution of spontaneous EPSC intervals in control (blue) and treated (red) conditions for 6 cells each. The cumulative probability curves differ clearly (Mann-Whitney $U$ test; $p=5.59 \times 10^{-169}$ ), with their intervals at the 0.5 cumulative probability being $465.7 \mathrm{~ms}$ versus $242 \mathrm{~ms}$ for control and PLX treatment, respectively.

resulting in an almost doubling of spontaneous EPSCs with PLX3397 treatment (PLX3397 vs control: $3.58 \pm 0.89 \mathrm{~Hz}$ vs $1.90 \pm 0.69 \mathrm{~Hz}[n=6$ example cells each]). We quantified this change in terms of EPSC interval measurements and find that the cumulative probability curves differ, with their intervals at the 0.5 cumulative probability being $465.7 \mathrm{~ms}$ versus $242 \mathrm{~ms}$ for control and PLX3397 treatment, respectively (Fig. 2G; MannWhitney $U$ test; $p=5.59 \times 10^{-169}$ ). However, individual EPSC amplitudes remain unchanged (Fig. 2F). This suggests that microglia depletion induces increases in presynaptic inputs from local cortical circuits rather than changes in quantal content.

Synaptic inputs from local cortical networks were then mapped in detail by combining whole-cell patch-clamp recordings with
LSPS via glutamate uncaging. This photostimulation-based approach has unique advantages allowing for extensive mapping and quantitative analysis of local synaptic connections to individually recorded neurons (Kuhlman et al., 2013; Sun et al., 2016; Xu et al., 2016). As illustrated in Figure $3 A$, the LSPS approach involves first recording from a single neuron, then stimulating sequentially throughout a matrix of sites to generate action potentials from presynaptic neurons. The recording from the potential postsynaptic neuron allows one to determine whether there is actual synaptic input from that particular site and its synaptic strength. Photostimulation induces two distinguishable forms of excitatory responses that are separable by timing and response kinetics: (1) those that result from direct activation of the recorded 
A

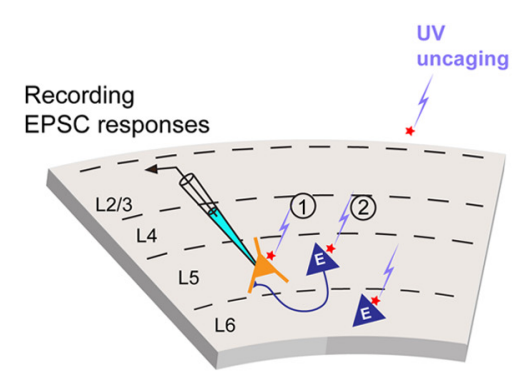

C

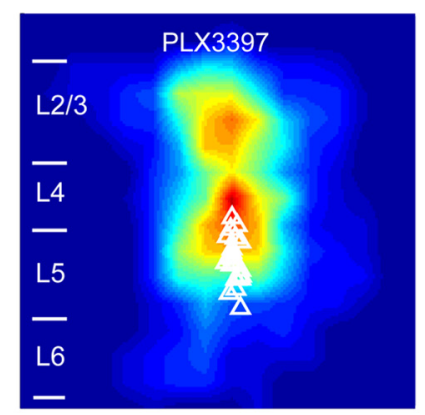

$\downarrow$ UV laser

(1)

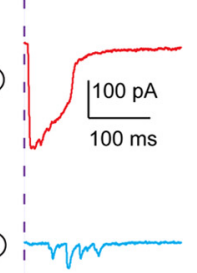

B

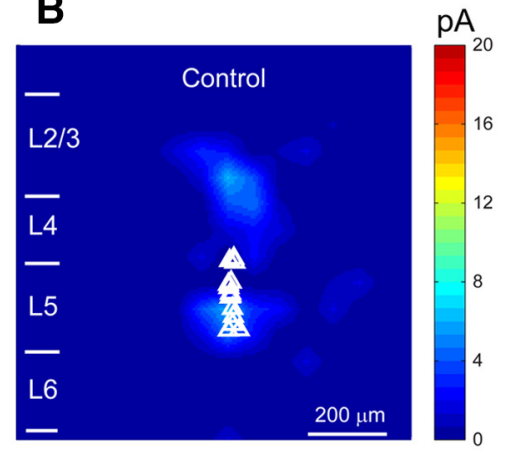

D

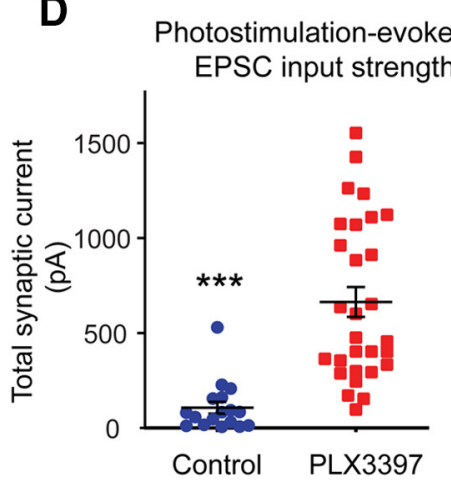

E

Total EPSC numbers

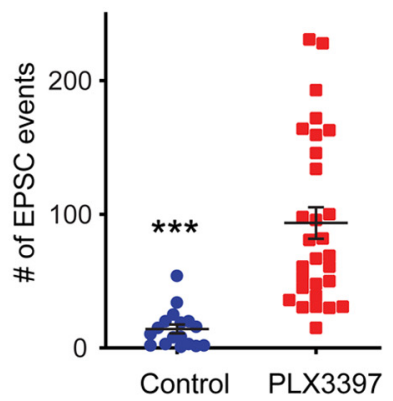

F

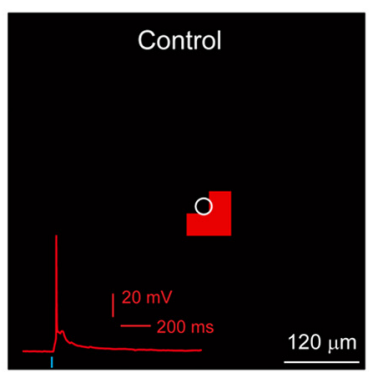

G

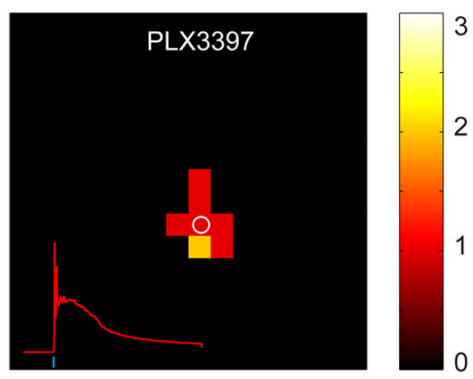

H

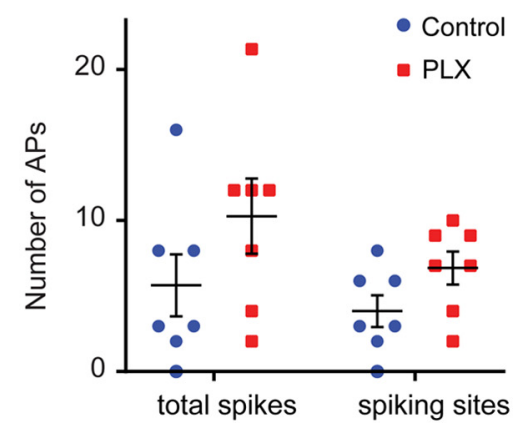

Figure 3. LSPS mapping in brain slices reveals enhanced local excitatory connections to excitatory pyramidal neurons in adult mouse visual cortex following 3-week-long PLX3397 treatment. A, Schematic of LSPS. During the experiment, a layer 5 pyramidal neuron (left) is recorded by patch clamping while stimulating its surrounding sites by short-duration UV glutamate uncaging to generate action potentials from potentially connected presynaptic neurons. Right, Example uncaging responses. Direct uncaging responses $(1$, red) are excluded from synaptic input analysis, and synaptically mediated EPSC responses (2, cyan) are plotted to show latency and amplitude characteristics of synaptic inputs from presynaptic neuronal spiking. $\boldsymbol{B}, \boldsymbol{C}$, The averaged photostimulation-evoked EPSC input maps from visual cortex in control mice $(n=17$ cells from 8 mice) and PLX3397 treatment mice ( $n=29$ cells from 10 mice), respectively. D, Average strengths of summed EPSC inputs in mice treated with PLX3397 ( $n=29$ cells) increase significantly compared with control cells $\left(n=17\right.$ cells) in adult mice $\left(p=1.48 \times 10^{-6}\right)$. $\boldsymbol{E}$, The numbers of total EPSC input events per cell for each group differ significantly (control vs PLX3397, $p=3.97 \times 10^{-7}$ ). Data are mean \pm SEM. $* * * p<0.001$ (Mann-Whitney $U$ tests). $\boldsymbol{F}-\boldsymbol{H}$, Spiking excitation profiles of excitatory neurons measured by glutamate uncaging show a trend of increase in excitability for the recorded cells in PLX3397-treated cortex ( $n=7$ cells from 4 mice), compared with controls ( $n=7$ cells from 4 mice; $p=0.17$ for total spikes; $p=0.085$ for spiking sites, Mann-Whitney $U$ test).

neuron's glutamate receptors and (2) synaptically mediated responses (EPSCs) resulting from the suprathreshold activation of presynaptic excitatory neurons (Fig. $3 A$, right). Responses that occur within $10 \mathrm{~ms}$ of laser pulse onset are considered direct; these responses are kinetically distinct and occur immediately after glutamate uncaging. Synaptic currents with such short latencies are not possible because they would have to occur before the generation of action potentials in photostimulated neurons.

Strikingly, V1 layer 5 pyramidal neurons in the PLX3397treated brain exhibit significantly enhanced local excitatory cortical connections with stronger EPSC amplitudes and a larger number of EPSC events compared with recordings from the control microglia intact brain. The group-averaged input maps pooled from the excitatory cells of microgliadepleted and control V1 display strikingly high-magnitude differences (Fig. $3 C$ vs Fig. $3 B$ ). The mean total ESPCs input per cell for adult mice are quadrupled in the absence of microglia (Fig. 3D; control vs PLX3397, $106.62 \pm 31.42 \mathrm{pA}$ $[n=17$ cells $]$ vs $663.68 \pm 78.42 \mathrm{pA}[n=29$ cells $]$, mean \pm SEM; $p=1.48 \times 10^{-6}$; Mann-Whitney $U$ test). Consistently, the numbers of total EPSC inputs per cell also differ with PLX3397 treatment, averaging 93.59 \pm 11.72 EPSC events $(n=29$ cells) versus $14.26 \pm 3.41$ events in controls $(n=17$ cells) (Fig. $3 E ; p=3.97 \times 10^{-7}$, Mann-Whitney $U$ test). 

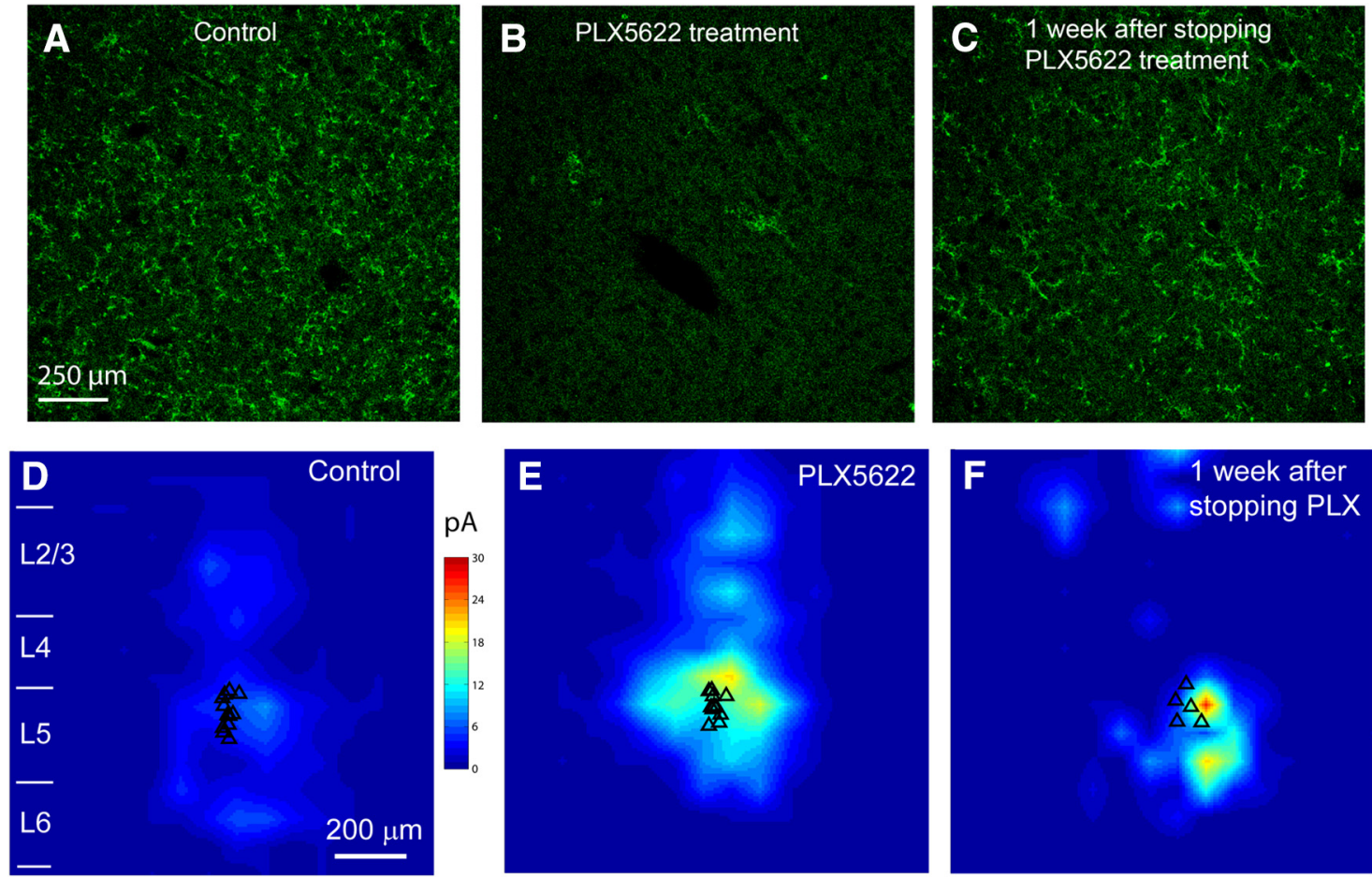

G

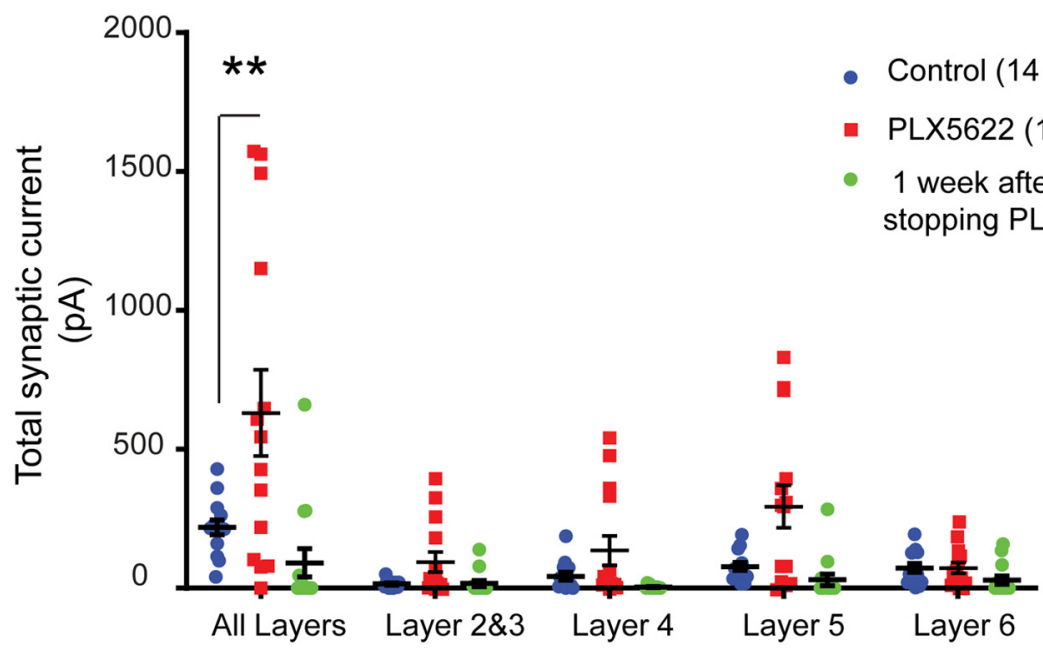

Figure 4. Microglial removal by a second CSFR1 inhibitor, PLX5622, shows similar effects on excitatory circuit connectivity, compared with the PLX3397 treatment. A-C, Example cortical images of IBA1 staining of microglia in control cortex, PLX5622-treated cortex, and the cortex repopulated with microglia 1 week after stopping PLX5622 treatment. D-F, The averaged photostimulation-evoked EPSC input maps of visual cortex in control mice in $\boldsymbol{D}$, PLX5622 treatment mice in $\boldsymbol{E}$, and repopulated cortex in $\boldsymbol{F}$. $\boldsymbol{G}$, Photostimulation-evoked EPSC inputs of recorded layer 5 excitatory neurons differ significantly between control ( $n=14$ cells from 4 mice) and 3-week-long PLX5622-treated groups ( $n=13$ cells from 4 mice) ( $p=0.027$; Mann-Whitney $U$ test). Also, the enhanced cortical connectivity of layer 5 excitatory cells ( $n=5$ cells from 4 mice) is reversed with microglia repopulation at a week after stopping PLX5622 treatment, as the total inputs of these cells did not differ from control cells in adult cortex $(p=0.559)$. Data are plotted as mean \pm SEM. $* * p<0.05$. In addition to all-layers inputs, cortical layer specific inputs are also plotted.

As PLX3397 inhibits not only CSF1R (leading to microglial depletion), but also c-kit (a related receptor), we sought to confirm these findings with the specific CSF1R inhibitor PLX5622 (Spangenberg et al., 2019). Notably, once microglia have been eliminated, withdrawal of the CSF1R inhibitor results in the rapid repopulation of the entire brain with new microglial cells (Elmore et al., 2014). Adult mice were treated with PLX5622 starting at 8-10 weeks of age, and adult brain slices were prepared and recorded 3-4 weeks after treatment. An additional group of mice were treated with PLX5622 for the same 3 to 4 week period, followed by PLX5622 withdrawal to stimulate repopulation. Microglia depletion and repopulation are confirmed using immunostaining for IBA1 (Fig. $4 A-C)$. In accordance with the PLX3397-treated mice, photostimulation-evoked EPSC inputs of recorded layer 5 excitatory neurons are significantly higher in PLX5622-treated microgliadepleted brains compared with age-matched controls (Fig. $4 D$, $E, G ; n=14$ and $n=13$ cells for PLX5622 and control, respectively; $p=0.027$, Mann-Whitney $U$ test). Notably, once microglia repopulate the brain following the cessation of CSF1R inhibitor treatment, these effects are reversed as the total synaptic input current in layer 5 excitatory cells ( $n=5$ cells) returns to control adult levels (Fig. $4 D, F, G ; p=0.559$, Mann-Whitney $U$ test). 
A
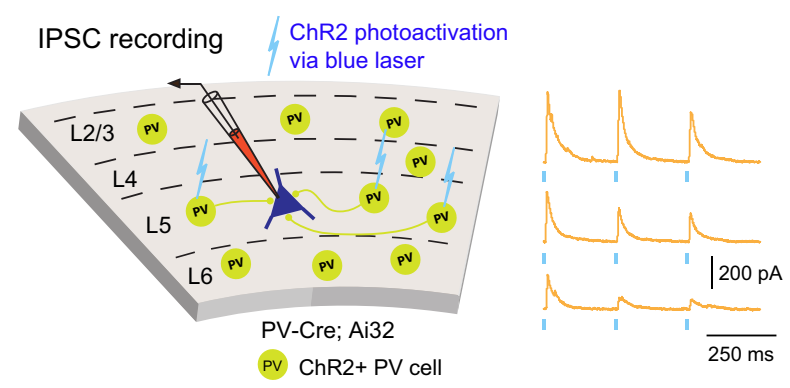

B

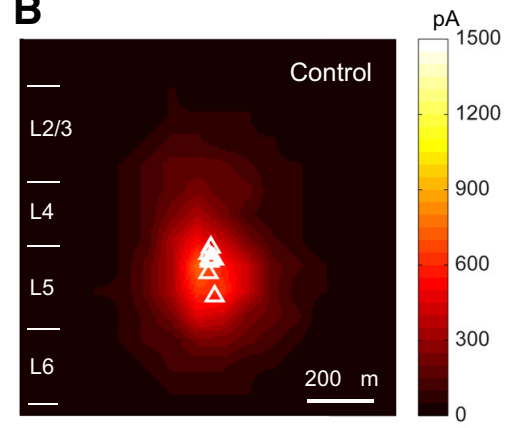

C

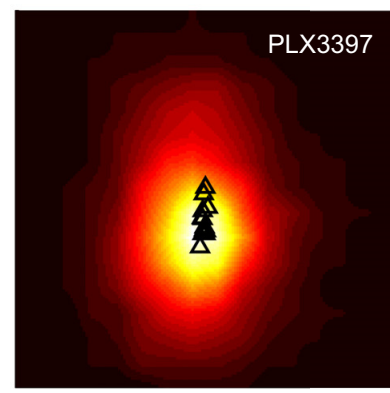

D

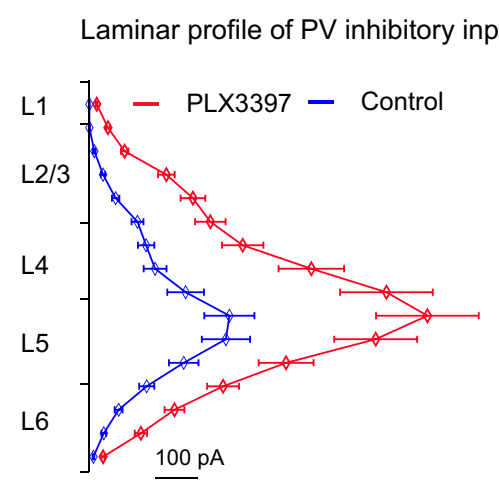

E

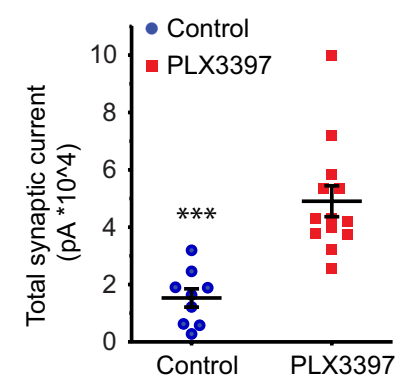

F

Total IPSC numbers across layers

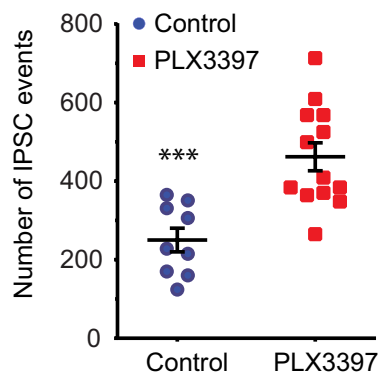

Figure 5. Microglia depletion with PLX3397 increases PV-specific inhibitory inputs to excitatory neurons in mouse visual cortex as assessed by ChR2-mediated circuit mapping. $\boldsymbol{A}$, Illustration of IPSC recordings by optogenetically evoking PV inhibitory inputs to a layer 5 pyramidal neuron in PV-Cre;Ai32 mice. The IPSCs from three locations of ChR2 photoactivation are shown on the right. Short blue vertical lines beneath the yellow response traces indicate $0.25 \mathrm{~ms}$ blue laser stimulation. $\boldsymbol{B}$, Average IPSCs input maps $(n=9$ cells from 4 mice) in control mice. $\boldsymbol{C}$, Average IPSC input maps ( $n=13$ cells from 4 mice) in PLX3397-treated mice. D, Plot of IPSCs from PV interneurons across the entire L1-L6 visual cortical layers for control mice (blue curve) and PLX3397 mice (red curve). $\boldsymbol{E}$, Average ChR2 photoactivation-evoked IPSC input strength in the PLX3397 treatment group is 3 times greater than in the control group $\left(p=1.4 \times 10^{-4}\right.$, Mann-Whitney $U$ test). $\boldsymbol{F}$, The average total IPSC event number in the PLX3397 treatment group is 2 times higher than in the control group $\left(p=6.6 \times 10^{-4}\right)$. Data are mean \pm SEM. $* * * p<0.001$.

\section{Microglial depletion increases PV-specific inhibitory inputs to cortical excitatory neurons}

Previous data reveal epileptiform activity and spontaneous seizures in mice deficient in C1q, a complement cascade protein that contributes to synaptic pruning (Chu et al., 2010). Here we show that microglial depletion increases excitatory connectivity yet does not evoke notably abnormal behaviors (i.e., seizures) in adult mice (Elmore et al., 2014; Spangenberg et al., 2019). The is consistent with our experiment finding that, while enhanced excitatory connections to layer 5 excitatory neurons may translate into increased excitability assessed with neuronal spike excitation profiles mapped by photostimulation (Fig. $3 F-H$ ), the overall excitation leading to neuronal spikes does not change substantially compared with control cells $(n=7$ cells each; $p=0.17$ for total spikes; $p=0.085$ for spiking sites, MannWhitney $U$ test). This suggests that the corresponding inhibitory connectivity must scale appropriately to functionally balance excitatory connectivity in the process of microglia depletion. PV interneurons are the most numerous inhibitory neurons in the cortex and their perisomatic inhibition of excitatory neurons has great impact in modulating cortical excitation (Cardin et al., 2009; Baohan et al., 2016). Given that microglia depletion upregulates PV expression in the visual cortex (Fig. 1G,I), we determined whether microglia depletion modulates inhibitory connections to excitatory neurons by optogenetically evoking PV-cell-specific inhibitory inputs to excitatory pyramidal neurons (Fig. 5A). We crossed PV-Cre mice to Cre-dependent ChR2 reporter mice
(Ai32) to direct ChR2 expression in PV neurons only. Sixweek-old PV-Cre;Ai32 mice were treated with PLX3397 or control chow for 3 weeks, and acute slices prepared. Direct $\mathrm{PV}^{+}$inhibitory connections to pyramidal neurons were mapped by ChR2 photoactivation of somatic spiking of presynaptic PV inhibitory neurons (Sun et al., 2016). Once again, V1 layer 5 excitatory neurons were recorded; their $\mathrm{PV}^{+}$specific inhibitory inputs were mapped with ChR2 photoactivation throughout the cortical site matrix. Representative examples of ChR2-photoactivation-evoked response traces, with the cell held at $5 \mathrm{mV}$ in voltage-clamp mode to detect outward IPSCs, are shown (Fig. 5A). Photoactivation reveals that excitatory pyramidal neurons receive greater $\mathrm{PV}^{+}$inhibitory inputs in the microglia-deleted brains (Fig. $5 C ; n=13$ cells) compared with age-matched control cells in microglia intact brains (Fig. $5 B ; n=9$ cells). The total amount of IPSCs per cell significantly increases threefold in treated mice, from $\sim 1.5 \times$ $10^{4} \mathrm{pA}$ to $\sim 4.9 \times 10^{4} \mathrm{pA}$ (Fig. $5 D, E ; p=1.4 \times 10^{-4}$, MannWhitney $U$ test). The number of IPSC events per cell significantly increases by a factor of 2, from 250 IPSC events to 462 in treated mice (Fig. 5F; $p=6.6 \times 10^{-4}$, Mann-Whitney $U$ test). These results show that, during normal development, microglia modulate both excitatory and inhibitory connections within the visual cortex, but that the effect on excitatory connections may be greater (i.e., a fourfold increase in glutamatergic inputs vs a twofold increase in $\mathrm{PV}^{+}$inhibitory inputs). Alternatively, this may be because of differences in excitatory versus inhibitory synaptic integration in recorded neurons. 
A

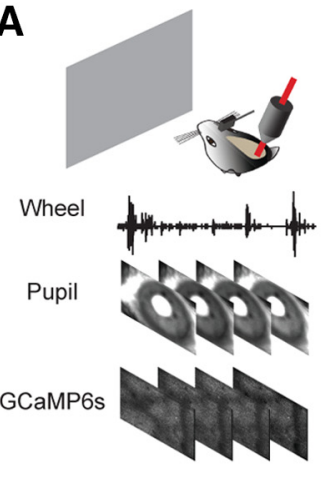

B

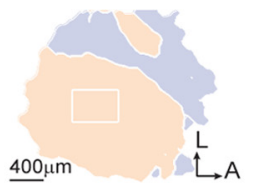

C

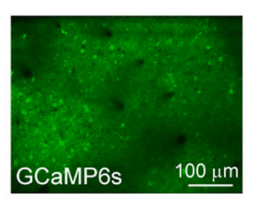

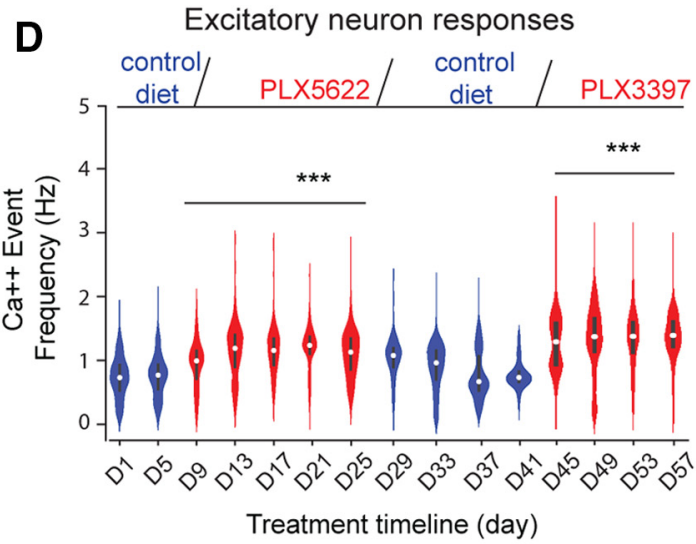

E

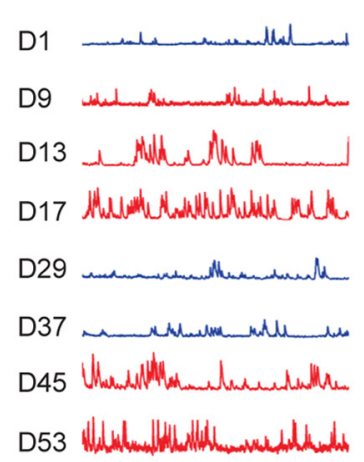

Cell 2

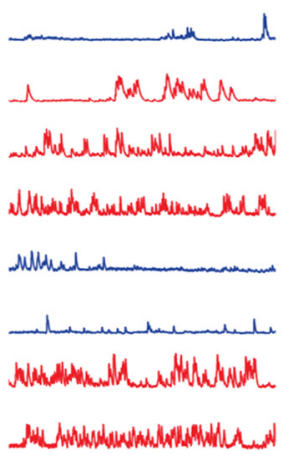

Cell 3

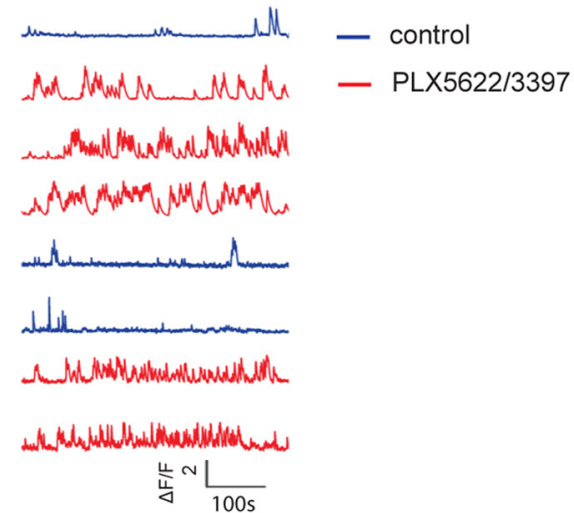

Figure 6. In vivo two-photon calcium imaging reveals that microglia depletion enhances V1 excitatory neuron activity. $\boldsymbol{A}$, A schematic illustration of in vivo two-photon imaging setup for a head-fixed awake mouse. During recording sessions, GCaMP6s calcium signals were recorded from V1 excitatory neurons; wheel tracking and pupil sizes were recorded simultaneously to determine animal running and eye movements. $\boldsymbol{B}$, A color-coded visual field map of visual cortex (left) is mapped before two-photon imaging. The imaging area (the white rectangle) is located within binocular V1. C, A z-projection image from the top surface view of the mouse cortex expressing GCaMP6s in excitatory neurons. $\boldsymbol{D}$, The violin plot graph of average calcium event frequencies of excitatory neurons (1144-1564 neurons from 5 mice; violin plot with median \pm SEM) as the mice viewed a gray screen during different conditions, including $5 \mathrm{~d}$ of the control condition, 3 weeks of depletion of microglia with the PLX5622 treatment, 2 weeks of microglia recovery, and 2 weeks of microglia redepletion with PLX3397. Top, Experimental timeline. Blue violins represent control or microglia repopulation groups. Red violins represent microglia depletion conditions. Neural activity was recorded every $4 \mathrm{~d}$, and was compared with neural activity on day 1 (D1) of control condition. Linear mixed-effects model analysis, overall: $p=5.19 \times 10^{-31}$. *** $p<0.001$. $\boldsymbol{E}$, Example calcium activity traces of three representative V1 excitatory neurons under control, microglia depletion, microglia repopulation, and microglia redepletion conditions. Blue traces represent calcium activity under control or microglia repopulation condition. Red traces represent activity under microglia depletion conditions.

\section{Microglia depletion enhances both excitatory and PV inhibitory population neuron activity in vivo}

Having demonstrated that microglial depletion dramatically increases both excitatory and inhibitory connectivity in the adult mouse visual cortex using acute slice preparations, we set about determining the large-scale neural circuit effects in vivo in awake mice. To accomplish this, we placed imaging windows in 16-week-old CaMK2-tTA;tetO-GCaMP6s mice that express GCaMP6s in excitatory neurons (Wekselblatt et al., 2016), to allow for subsequent two-photon calcium imaging of large population of neurons in the binocular V1 area for 1 month or more. Longitudinal recording in awake, head-fixed mice was performed using the conditions depicted in Figures 6 and 7, with microglia-depleting paradigms with either PLX5622 or PLX3397. Withdrawal of CSF1R inhibitor-containing chow (i.e., switches to control chow) was used to stimulate repopulation of the microglial tissue. The boundary of V1 cortex (Fig. $6 B$, yellow) was identified using visual field mapping stimuli (Kalatsky and Stryker, 2003). Mice viewed a gray screen with $20 \mathrm{~cd} / \mathrm{m}^{2}$ luminance (Fig. 6A) through the contralateral eye for $15 \mathrm{~min}$, and GCaMP6s imaging of neural activity was performed using two-photon microscopy from layer $2 / 3$ in binocular V1. Wheel movements, eye movements, and pupil sizes were collected simultaneously to monitor animal behaviors. The timeline in days is shown at the bottom of Figure $6 D$ and corresponds to the experimental condition shown at the top of Figure $6 D$. Briefly, the animals were fed with control chow for recording at the control condition ( $5 \mathrm{~d}$ starting at 16 weeks of age), followed by PLX5622 chow to deplete microglia for 3 weeks, and then replacement with control chow for 2 weeks, resulting in microglial repopulation (Elmore et al., 2014). As we were interested in comparing the in vivo effects of both CSF1R inhibitors, following this period of repopulation, microglia were subsequently redepleted with PLX3397 for 2 weeks in additional experiments.

The neural activities of excitatory pyramidal neurons located in layer 2/3 of V1 from 5 mice were recorded every $4 \mathrm{~d}$. Calcium signal traces of representative excitatory neurons under control, microglia depletion, and microglia repopulation treatment conditions are shown in Figure $6 E$. Blue traces are used to depict calcium activity signals in the control or microglia repopulation conditions, and red traces are for calcium activity signals with 
A

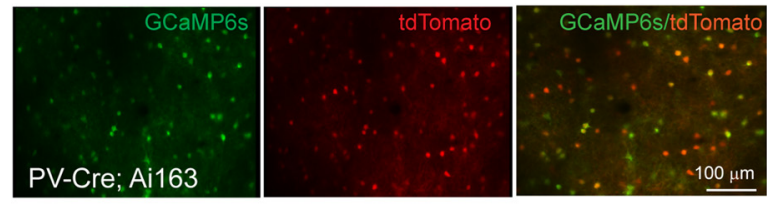

PV neuron responses
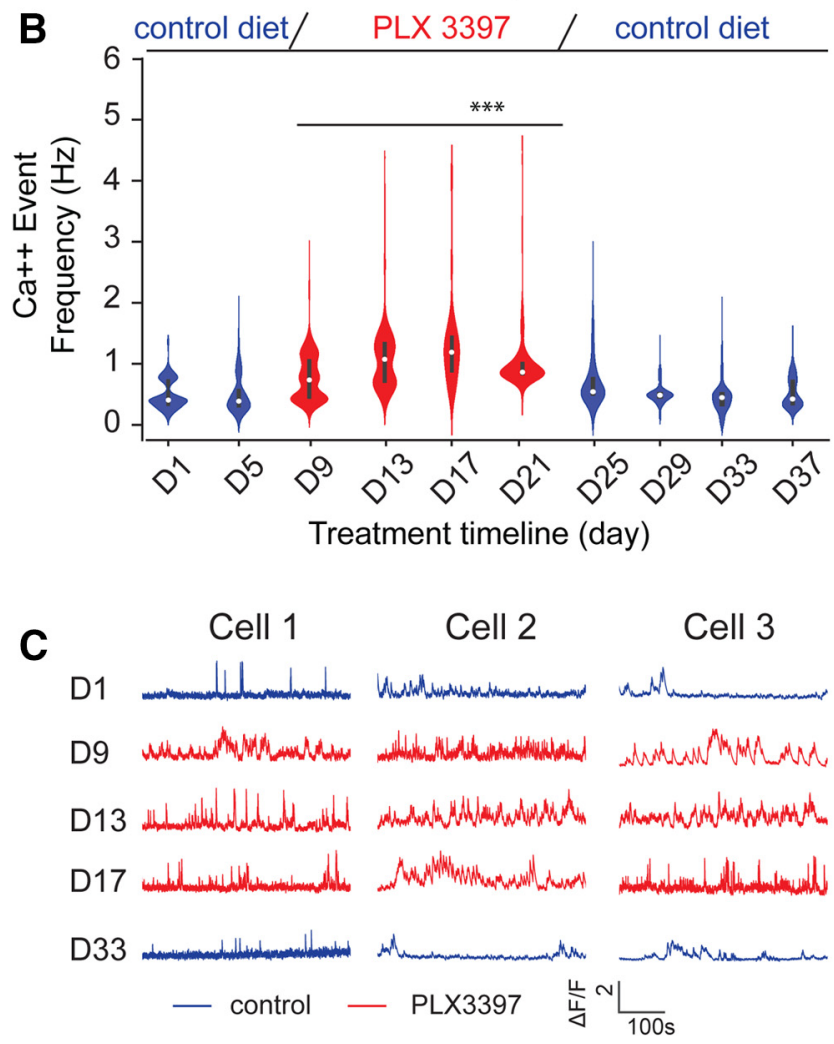

Figure 7. In vivo two-photon calcium imaging reveals that microglia depletion enhances PV interneuron activity. A, A representative cortical image of PV-Cre;Ai163 mice with PV interneurons (tdTomato, red) coexpressing GCaMP6s (green). $\boldsymbol{B}$, A violin plot graph of average calcium event frequencies of PV inhibitory neurons (violin plot with median \pm SEM) across the control chow condition (blue), microglia depletion by PLX3397 treatment (red), and microglia repopulation (blue). A total of 541-733 neurons from 3 mice are included for analysis. Neural activity was recorded every $4 \mathrm{~d}$ and was compared with neural activity on day 1 (D1) of control condition. Linear mixed-effects model analysis, overall: $p=6.27 \times 10^{-4}$. $* * * p<0.001$. C, Example calcium activity traces of three PV neurons from PV-Cre;Ai163 mice across control, microglia depletion, and microglia repopulation conditions.

microglial depletion. The neural activity with either PLX5622 or PLX3397 treatment (Fig. 6E, red traces) is significantly stronger than that measured in control chow animals (Fig. 6E, blue traces). Average calcium event frequencies of excitatory neuron across different conditions are plotted in Figure $6 D$. The neural activity for control chow and recovery at each time point is significantly lower than that measured in PLX5622/PLX3397 treatment animals (overall $p=0.001$, Kruskal-Wallis test). Next, neural activity of each day was compared with baseline activity of control day 1 (D1). The average calcium event frequency increases significantly with microglia depletion under PLX5622 treatments (vs D1, D9 $p=5.194 \times 10^{-53}$, D13 $p=1.187 \times 10^{-138}$, D17 $p=1.45 \times 10^{-137}, \mathrm{D} 21 p=3.74 \times 10^{-191}, \mathrm{D} 25 p=6.66 \times$ $10^{-137}$, Mann-Whitney $U$ test, adjusted for multiple comparisons). Microglial repopulation returns the excitatory neuronal activity to baseline level after $12 \mathrm{~d}$ of recovery time (D1 vs D37: $0.74 \pm 0.01$ vs $0.77 \pm 0.01 \mathrm{~Hz}$, mean \pm SEM; vs D1, D29 $p=6.658 \times 10^{-114}, \mathrm{D} 33 p=1.25 \times 10^{-43}, \mathrm{D} 37 p=0.768, \mathrm{D} 41$ $p=0.060$, Mann-Whiney $U$ test). Excitatory neural activity then subsequently increases by $\sim 20 \%$ with microglial redepletion evoked by $14 \mathrm{~d}$ of PLX3397 treatment (vs D1, D45 $p=1.44 \times 10^{-129}$, D49 $p=1.24 \times 10^{-146}$, D53 $p=7.07 \times 10^{-145}$, D57 $p=3.18 \times 10^{-212}$, Mann Whiney $U$ test). We identified consistent differences using a linear mixed-effects model analysis with control chow and PLX5622/PLX3397 treatment groups as a fixed effect and mouse identity as a random effect: overall, $p=5.19 \times 10^{-31} ; \mathrm{D} 5, p=0.148$; D9, $p=3.37 \times 10^{-43} ; \quad$ D13,$p=3.44 \times 10^{-172} ; \quad$ D17, $p=2.74 \times$ $10^{-130} ; \mathrm{D} 21, \quad p=1.96 \times 10^{-198} ; \mathrm{D} 25, \quad p=2.25 \times 10^{-162} ; \mathrm{D} 29$, $p=8.86 \times 10^{-84} ; \quad \mathrm{D} 33, \quad p=1.55 \times 10^{-33} ; \mathrm{D} 37, \quad p=0.352 ; \mathrm{D} 41$, $p=0.064 ; \mathrm{D} 45, p=5.63 \times 10^{-55} ; \mathrm{D} 49, p=1.37 \times 10^{-128} ; \mathrm{D} 53, p=$ $1.22 \times 10^{-83} ; \mathrm{D} 57, p=7.21 \times 10^{-119}$ ( $p$ values adjusted for multiple comparison). Thus, in vivo two-photon recordings from excitatory neurons show that microglial depletion induces sustained increases of population excitatory neuron activity at single-cell resolution.

Our slice-based mapping results show that microglia regulate both excitatory and PV-inhibitory connections to excitatory neurons in the visual cortex. We further tested how microglial depletion regulates PV interneuron activity in vivo by performing two-photon longitudinal recordings from V1 layer 2/3 PV neurons. Calcium activity of PV cells was measured from PV-Cre; Ail63 mice, in which the genetically encoded calcium indicator GCaMP6s is selectively expressed in PV neurons (Daigle et al., 2018) (Fig. 7A). Similar to excitatory neurons, the activities of $\mathrm{PV}$ interneurons were recorded every $4 \mathrm{~d}$ during control conditions, microglia depletion by PLX3397 treatment conditions, and microglia repopulation conditions (Fig. $7 B, C$ ). As the neural activity effects of PLX5622 and PLX3397 are similar, we only used PLX3397 treatment for the PV-Cre;Ai163 mice. Figure 7C shows representative example calcium activities of $\mathrm{PV}$ neurons under control, microglia depletion, and microglia recovery conditions. The average calcium event frequency during the PLX3397 treatment condition in Figure $7 B$ is significantly different from the control condition $(p=0.001$, Kruskal Wallis Test). Mann-Whitney $U$ test (adjusted for multiple comparisons) were used to compare neural activities with control days. Consistent with enhanced activities of excitatory neurons, $\mathrm{PV}$ interneurons show elevated activities in the absence of microglia (vs D1, D9 $p=2.52 \times 10^{-54}$, D13 $p=2.68 \times$ $\left.10^{-102}, \mathrm{D} 17 p=2.06 \times 10^{-99}, \mathrm{D} 21 p=3.23 \times 10^{-183}\right)$. PV cell activities are then restored to baseline activity with subsequent microglial repopulation (vs D1, D25 $p=2.32 \times 10^{-26}$, D29 $\left.p=3.56 \times 10^{-9}, \mathrm{D} 33 p=0.280, \mathrm{D} 37 p=0.139\right)$. Furthermore, the effect is confirmed by a linear mixed-effects model analysis, which used control chow and PLX3397 treatment groups as a fixed effect and mouse identity as a random effect: in Figure $7 B$, overall, $p=6.27 \times 10^{-4}$; versus D1, D5 $p=0.849$; D9 $p=3.43 \times 10^{-24} ;$ D13 $p=6.93 \times 10^{-61} ;$ D17 $p=1.95 \times$ $10^{-168} ; \mathrm{D} 21 p=3.78 \times 10^{-157} ; \mathrm{D} 25 p=7.32 \times 10^{-21} ; \mathrm{D} 29 p=$ $5.83 \times 10^{-4} ; \mathrm{D} 33 p=0.241 ; \mathrm{D} 37 p=0.417$ ( $p$ values adjusted for multiple comparison). Increased activities of PV cells indicate that microglia depletion enhances in vivo neural activity of PV inhibitory interneurons in the visual cortex, which is consistent with our circuit mapping data and the absence of seizures following CSF1R inhibitor treatment. Together, our results establish microglial regulation of activities of both excitatory neurons and PV inhibitory neurons in adult cortex in vivo. 


\section{Discussion}

Evidence that microglia shape synaptic architecture has emerged in the last few decades. However, the significant question of whether and how they regulate adult cortical circuit connections remains open. In the present study, we address our hypothesis that microglia regulate neural circuit connectivity and functional activity in the adult brain using CSF1R inhibitors for dependable in vivo microglial depletion (Green et al., 2020). While most myeloid lineage cells express CSF1R, the microglia (and other CNS macrophages) are uniquely sensitive to CSF1R inhibitors and are the only cells in the brain that express CSF1R. Thus, CSF1R inhibitors offer an effective approach to manipulate the microglial tissue without effects on other brain cell types, and can be used to both deplete microglia, but also to repopulate the entire microglial tissue on their withdrawal. We used an integrative series of experiments combining anatomical, ex vivo and in vivo neural circuit mapping experiments to study the roles of microglia in neural circuit connectivity. Our new findings extend previous key findings from the developing brain, where microglia are known to prune normal neuronal connectivity. Our findings offer new insights in microglia-mediated sculpting of neural connectivity and subsequent regulation of functional activity in adult cortex.

Anatomically, we find that chronic microglial depletion for 3 weeks leads to increased densities and intensities of PNNs in adult cortex. This is supported by recent evidence of microglial remodeling of the extracellular matrix (Nguyen et al., 2020). Functionally, we find that microglia depletion dramatically increases both excitatory and inhibitory synaptic connections to excitatory cortical neurons without altering their intrinsic electrophysiological properties. Increased synaptic connectivity after transient microglia depletion is reversed by microglial repopulation after cessation of CSF1R inhibitor treatment within a week; thus, microglia depletion and repopulation modulate neural activity in the adult cortex. The physiological implications of microglial removal are addressed by in vivo calcium imaging of large populations of visual cortical neurons that shows enhanced neural activities of both excitatory neurons and PV interneurons. Reversibility of a pharmacological approach in adults does not have the developmental abnormality issue of a purely genetic approach, as exhibited in the mice deficient in C1q KO mice (Chu et al., 2010) (see below).

Our findings are highly interesting in light of the developmental literature. During development, there is an overproduction of synapses in the brain, which must be pruned back to refine and establish appropriate neuronal connectivity. Multiple groups have shown that microglia are capable of phagocytosing presynaptic and postsynaptic inputs (Kettenmann et al., 2013; Szepesi et al., 2018). CX3CR1 deficiency leads to a transient reduction in microglia during early postnatal development that results in increased numbers of PSD-95 puncta and dendritic spines (Paolicelli et al., 2011) and leads to long-term synaptic connectivity changes that persist into P40 (Zhan et al., 2014). Our new data establish that microglial depletion effectively regulates adult cortical connectivity and functional neural activity in similar ways as observed during the early postnatal development. Recent studies of eliminating microglia from adult mouse brains have provided supporting evidence. Acute microglial depletion via targeted diphtheria toxin leads to changes in dendritic spine dynamics (Parkhurst et al., 2013). Our prior studies show that chronic microglia depletion by CSF1R inhibitors leads to robust increases in dendritic spine densities (Elmore et al., 2014; Rice et al., 2015, 2017), as well as increases in synaptic puncta.
Furthermore, mice deficient for C1q or the downstream complement protein $\mathrm{C} 3$ exhibit excess synapses as a result of a pruning failure (Stevens et al., 2007; Chu et al., 2010). These mice do show spontaneous seizures because of increased excitatory cortical connections (without altered inhibitory connectivity). However, our results with adult chronic microglia depletion with CSF1R inhibitors are distinguishable from $\mathrm{Clq}$ and $\mathrm{C} 3$ genetic knock-outs CSF1R inhibitor treatment increases both excitatory and inhibitory synaptic connections to excitatory cortical neurons, and adult microglia-depleted animals do not exhibit seizure behaviors. Counter to possible deficits in cognition, we have reported improvements in spatial memory performance in adult microgliadepleted mice (Elmore et al., 2014; Rice et al., 2015; Spangenberg et al., 2019). This is supported further by recent findings that microglia mediate forgetting of memories (Wang et al., 2020).

Furthermore, the question of whether microglia modulate inhibitory synaptic connections has not been directly examined previously. Using an optogenetic approach, we show that, following microglia depletion, $\mathrm{PV}^{+}$inhibitory synaptic connections to excitatory neurons are increased. This demonstration establishes that microglia can sculpt both excitatory and inhibitory synaptic connections in cortical circuits. We find also that, in the absence of microglia in adult animals, PV neurons exhibit enhanced activity in vivo, which by way of inhibitory/excitatory balance, accounts for a lack of seizure behavioral phenotypes concomitant with increased excitatory neuronal activity.

A major highlight, alongside increased circuit connectivity, is that robust changes in PNNs follow microglial depletion. PNNs surround certain neuronal subtypes, particularly PV inhibitory neurons, and are usually associated with a reduction in plasticity (Carulli et al., 2010; Conant et al., 2010; Sorg et al., 2016), and are seen as restricting synapse growth (Dityatev et al., 2007). As PNNs also form around somatostatin-positive neurons in the hypothalamic arcuate nucleus (Mirzadeh et al., 2019), we will be interested in examining the microglial removal impact on somatostatin-positive interneurons in future studies. Breakdown of PNNs with chondroitinase- $\mathrm{ABC}$ is known to restore plasticity (Pizzorusso et al., 2002; Lensjo et al., 2017). The increase in PNNs following microglial depletion may be a compensatory reaction to increased synaptic inputs. In vitro and in vivo experiments show that PNNs form in response to neuronal activity (Pizzorusso et al., 2002; Sorg et al., 2016). Similarly, increased PV expression has been associated with increased neuronal activity (Dityatev et al., 2007; Donato et al., 2013). This is consistent with our present findings by in vivo two-photon imaging results. In conclusion, our study establishes that microglia regulate neural circuit connectivity and activity by modifying both excitatory and inhibitory synaptic connections to excitatory neurons in adult cortex. These results reveal new potential avenues to enhance adult neural plasticity, which can be applied to treat brain disorders and injuries, including both neurodegenerative diseases and neuropsychiatric disorders.

\section{References}

Baohan A, Ikrar T, Tring E, Xu X, Trachtenberg JT (2016) Pten and EphB4 regulate the establishment of perisomatic inhibition in mouse visual cortex. Nat Commun 7:12829.

Brückner G, Grosche J, Schmidt S, Hartig W, Margolis RU, Delpech B, Seidenbecher CI, Czaniera R, Schachner M (2000) Postnatal development of perineuronal nets in wild-type mice and in a mutant deficient in tenascin-R. J Comp Neurol 428:616-629.

Cardin JA, Carlen M, Meletis K, Knoblich U, Zhang F, Deisseroth K, Tsai LH, Moore CI (2009) Driving fast-spiking cells induces gamma rhythm and controls sensory responses. Nature 459:663-667. 
Carulli D, Pizzorusso T, Kwok JC, Putignano E, Poli A, Forostyak S, Andrews MR, Deepa SS, Glant TT, Fawcett JW (2010) Animals lacking link protein have attenuated perineuronal nets and persistent plasticity. Brain 133:2331-2347.

Chu Y, Jin X, Parada I, Pesic A, Stevens B, Barres B, Prince DA (2010) Enhanced synaptic connectivity and epilepsy in C1q knockout mice. Proc Natl Acad Sci USA 107:7975-7980.

Colonna M, Butovsky O (2017) Microglia function in the central nervous system during health and neurodegeneration. Annu Rev Immunol 35:441468.

Conant K, Wang Y, Szklarczyk A, Dudak A, Mattson MP, Lim ST (2010) Matrix metalloproteinase-dependent shedding of intercellular adhesion molecule-5 occurs with long-term potentiation. Neuroscience 166:508521.

Crapser JD, Ochaba J, Soni N, Reidling JC, Thompson LM, Green KN (2020a) Microglial depletion prevents extracellular matrix changes and striatal volume reduction in a model of Huntington's disease. Brain 143:266-288.

Crapser JD, Spangenberg EE, Barahona RA, Arreola MA, Hohsfield LA, Green KN (2020b) Microglia facilitate loss of perineuronal nets in the Alzheimer's disease brain. EBioMedicine 58:102919.

Daigle TL, Madisen L, Hage TA, Valley MT, Knoblich U, Larsen RS, Takeno MM, Huang L, Gu H, Larsen R, Mills M, Bosma-Moody A, Siverts LA, Walker M, Graybuck LT, Yao Z, Fong O, Nguyen TN, Garren E, Lenz $\mathrm{GH}$, et al. (2018) A suite of transgenic driver and reporter mouse lines with enhanced brain-cell-type targeting and functionality. Cell 174:465480.e422.

Dityatev A, Brückner G, Dityateva G, Grosche J, Kleene R, Schachner M (2007) Activity-dependent formation and functions of chondroitin sulfate-rich extracellular matrix of perineuronal nets. Dev Neurobiol 67:570-588

Donato F, Rompani SB, Caroni P (2013) Parvalbumin-expressing basket-cell network plasticity induced by experience regulates adult learning. Nature 504:272-276.

Elmore MR, Najafi AR, Koike MA, Dagher NN, Spangenberg EE, Rice RA, Kitazawa M, Matusow B, Nguyen H, West BL, Green KN (2014) Colonystimulating factor 1 receptor signaling is necessary for microglia viability, unmasking a microglia progenitor cell in the adult brain. Neuron 82:380397.

Garrett ME, Nauhaus I, Marshel JH, Callaway EM (2014) Topography and areal organization of mouse visual cortex. J Neurosci 34:12587-12600.

Giovannucci A, Friedrich J, Gunn P, Kalfon J, Brown BL, Koay SA, Taxidis J, Najafi F, Gauthier JL, Zhou P, Khakh BS, Tank DW, Chklovskii DB, Pnevmatikakis EA (2019) CaImAn: an Open Source tool for scalable calcium imaging data analysis. Elife 8:e38173.

Green KN, Crapser JD, Hohsfield LA (2020) To kill a microglia: a case for CSF1R inhibitors. Trends Immunol 41:771-784.

Grieco SF, Qiao X, Zheng X, Liu Y, Chen L, Zhang H, Yu Z, Gavornik JP, Lai C, Gandhi SP, Holmes TC, Xu X (2020) Subanesthetic ketamine reactivates adult cortical plasticity to restore vision from amblyopia. Curr Biol 30:3591-3603.e8.

Hartig W, Brauer K, Brückner G (1992) Wisteria floribunda agglutininlabelled nets surround parvalbumin-containing neurons. Neuroreport 3:869-872

Ji K, Akgul G, Wollmuth LP, Tsirka SE (2013) Microglia actively regulate the number of functional synapses. PLoS One 8:e56293.

Kalatsky VA, Stryker MP (2003) New paradigm for optical imaging: temporally encoded maps of intrinsic signal. Neuron 38:529-545.

Kaplan ES, Cooke SF, Komorowski RW, Chubykin AA, Thomazeau A, Khibnik LA, Gavornik JP, Bear MF (2016) Contrasting roles for parvalbumin-expressing inhibitory neurons in two forms of adult visual cortical plasticity. Elife 5:e11450.

Kettenmann H, Kirchhoff F, Verkhratsky A (2013) Microglia: new roles for the synaptic stripper. Neuron 77:10-18.

Kuhlman SJ, Olivas ND, Tring E, Ikrar T, Xu X, Trachtenberg JT (2013) A disinhibitory microcircuit initiates critical-period plasticity in the visual cortex. Nature 501:543-546.

Lensjo KK, Lepperod ME, Dick G, Hafting T, Fyhn M (2017) Removal of perineuronal nets unlocks juvenile plasticity through network mechanisms of decreased inhibition and increased gamma activity. J Neurosci $37: 1269-1283$
Madisen L, Mao T, Koch H, Zhuo J-m, Berenyi A, Fujisawa S, A Hsu Y-W, Garcia AJ, Gu X, Zanella S, Kidney J, Gu H, Mao Y, Hooks BM, Boyden ES, Buzsáki G, Ramirez JM, Jones AR, Svoboda K, Han X et al. (2012) A toolbox of Cre-dependent optogenetic transgenic mice for light-induced activation and silencing. Nat Neurosci 15:793-802.

Mirzadeh Z, Alonge KM, Cabrales E, Herranz-Pérez V, Scarlett JM, Brown JM, Hassouna R, Matsen ME, Nguyen HT, Garcia-Verdugo JM, Zeltser LM, Schwartz MW (2019) Perineuronal net formation during the critical period for neuronal maturation in the hypothalamic arcuate nucleus. Nat Metab 1:212-221.

Moda-Sava RN, Murdock MH, Parekh PK, Fetcho RN, Huang BS, Huynh TN, Witztum J, Shaver DC, Rosenthal DL, Alway EJ (2019) Sustained rescue of prefrontal circuit dysfunction by antidepressant-induced spine formation. Science 364:eaat8078.

Nayak D, Roth TL, McGavern DB (2014) Microglia development and function. Annu Rev Immunol 32:367-402.

Nguyen PT, Dorman LC, Pan S, Vainchtein ID, Han RT, Nakao-Inoue H, Taloma SE, Barron JJ, Molofsky AB, Kheirbek MA, Molofsky AV (2020) Microglial remodeling of the extracellular matrix promotes synapse plasticity. Cell 182:388-403.e15.

Nimmerjahn A, Kirchhoff F, Helmchen F (2005) Resting microglial cells are highly dynamic surveillants of brain parenchyma in vivo. Science 308:1314-1318.

Olivas ND, Quintanar-Zilinskas V, Nenadic Z, Xu X (2012) Laminar circuit organization and response modulation in mouse visual cortex. Front Neural Circuits 6:70.

Paolicelli RC, Bolasco G, Pagani F, Maggi L, Scianni M, Panzanelli P, Giustetto M, Ferreira TA, Guiducci E, Dumas L, Ragozzino D, Gross CT (2011) Synaptic pruning by microglia is necessary for normal brain development. Science 333:1456-1458.

Parkhurst CN, Yang G, Ninan I, Savas JN, Yates JR, Lafaille JJ, Hempstead BL, Littman DR, Gan WB (2013) Microglia promote learning-dependent synapse formation through brain-derived neurotrophic factor. Cell 155:1596-1609.

Pizzorusso T, Medini P, Berardi N, Chierzi S, Fawcett JW, Maffei L (2002) Reactivation of ocular dominance plasticity in the adult visual cortex. Science 298:1248-1251.

Pnevmatikakis EA, Giovannucci A (2017) NoRMCorre: an online algorithm for piecewise rigid motion correction of calcium imaging data. J Neurosci Methods 291:83-94.

Pnevmatikakis EA, Soudry D, Gao Y, Machado TA, Merel J, Pfau D, Reardon T, Mu Y, Lacefield C, Yang W, Ahrens M, Bruno R, Jessell TM, Peterka DS, Yuste R, Paninski L (2016) Simultaneous denoising, deconvolution, and demixing of calcium imaging data. Neuron 89:285-299.

Rajendran L, Paolicelli RC (2018) Microglia-mediated synapse loss in Alzheimer's disease. J Neurosci 38:2911-2919.

Rice RA, Spangenberg EE, Yamate-Morgan H, Lee RJ, Arora RP, Hernandez MX, Tenner AJ, West BL, Green KN (2015) Elimination of microglia improves functional outcomes following extensive neuronal loss in the hippocampus. J Neurosci 35:9977-9989.

Rice RA, Pham J, Lee RJ, Najafi AR, West BL, Green KN (2017) Microglial repopulation resolves inflammation and promotes brain recovery after injury. Glia 65:931-944.

Schafer DP, Lehrman EK, Kautzman AG, Koyama R, Mardinly AR, Yamasaki R, Ransohoff RM, Greenberg ME, Barres BA, Stevens B (2012) Microglia sculpt postnatal neural circuits in an activity and complementdependent manner. Neuron 74:691-705.

Shi Y, Nenadic Z, Xu X (2010) Novel use of matched filtering for synaptic event detection and extraction. PLoS One 5:e15517.

Sorg BA, Berretta S, Blacktop JM, Fawcett JW, Kitagawa H, Kwok JC, Miquel M (2016) Casting a wide net: role of perineuronal nets in neural plasticity. J Neurosci 36:11459-11468.

Spangenberg E, Severson PL, Hohsfield LA, Crapser J, Zhang J, Burton EA, Zhang Y, Spevak W, Lin J, Phan NY, Habets G, Rymar A, Tsang G, Walters J, Nespi M, Singh P, Broome S, Ibrahim P, Zhang C, Bollag G, et al. (2019) Sustained microglial depletion with CSF1R inhibitor impairs parenchymal plaque development in an Alzheimer's disease model. Nat Commun 10:3758.

Spangenberg EE, Lee RJ, Najafi AR, Rice RA, Elmore MR, Blurton-Jones M, West BL, Green KN (2016) Eliminating microglia in Alzheimer's mice prevents neuronal loss without modulating amyloid-beta pathology. Brain 139:1265-1281. 
Stevens B, Allen NJ, Vazquez LE, Howell GR, Christopherson KS, Nouri N, Micheva KD, Mehalow AK, Huberman AD, Stafford B, Sher A, Litke AM, Lambris JD, Smith SJ, John SW, Barres BA (2007) The classical complement cascade mediates CNS synapse elimination. Cell 131:1164-1178.

Stobart JL, Ferrari KD, Barrett MJ, Gluck C, Stobart MJ, Zuend M, Weber B (2018a) Cortical circuit activity evokes rapid astrocyte calcium signals on a similar timescale to neurons. Neuron 98:726-735.e724.

Stobart JL, Ferrari KD, Barrett MJ, Stobart MJ, Looser ZJ, Saab AS, Weber B (2018b) Long-term in vivo calcium imaging of astrocytes reveals distinct cellular compartment responses to sensory stimulation. Cereb Cortex 28:184-198.

Sun Y, Ikrar T, Davis MF, Gong N, Zheng X, Luo ZD, Lai C, Mei L, Holmes TC, Gandhi SP, Xu X (2016) Neuregulin-1/ErbB4 signaling regulates visual cortical plasticity. Neuron 92:160-173.

Szepesi Z, Manouchehrian O, Bachiller S, Deierborg T (2018) Bidirectional microglia-neuron communication in health and disease. Front Cell Neurosci 12:323.

Tremblay ME, Stevens B, Sierra A, Wake H, Bessis A, Nimmerjahn A (2011) The role of microglia in the healthy brain. J Neurosci 31:16064-16069.

Wang C, Yue H, Hu Z, Shen Y, Ma J, Li J, Wang XD, Wang L, Sun B, Shi P, Wang L, Gu Y (2020) Microglia mediate forgetting via complement-dependent synaptic elimination. Science 367:688-694.
Wekselblatt JB, Flister ED, Piscopo DM, Niell CM (2016) Large-scale imaging of cortical dynamics during sensory perception and behavior. J Neurophysiol 115:2852-2866.

Xu X, Callaway EM (2009) Laminar specificity of functional input to distinct types of inhibitory cortical neurons. J Neurosci 29:70-85.

Xu X, Roby KD, Callaway EM (2010a) Immunochemical characterization of inhibitory mouse cortical neurons: Three chemically distinct classes of inhibitory cells. J Comp Neurol 518:389-404.

Xu X, Olivas ND, Levi R, Ikrar T, Nenadic Z (2010b) High precision and fast functional mapping of cortical circuitry through a combination of voltage sensitive dye imaging and laser scanning photostimulation. J Neurophysiol 103:2301-2312.

Xu X, Olivas ND, Ikrar T, Peng T, Holmes TC, Nie Q, Shi Y (2016) Primary visual cortex shows laminar-specific and balanced circuit organization of excitatory and inhibitory synaptic connectivity. J Physiol 594:1891-1910.

Zhan Y, Paolicelli RC, Sforazzini F, Weinhard L, Bolasco G, Pagani F, Vyssotski AL, Bifone A, Gozzi A, Ragozzino D, Gross CT (2014) Deficient neuron-microglia signaling results in impaired functional brain connectivity and social behavior. Nat Neurosci 17:400-406.

Zhuang J, Ng L, Williams D, Valley M, Li Y, Garrett M, Waters J (2017) An extended retinotopic map of mouse cortex. Elife 6:e18372. 